\title{
Code-timing synchronization in DS-CDMA systems using space-time diversity
}

\author{
Gonzalo Seco $^{\text {a }}$, Juan A. Fernández-Rubio ${ }^{\mathrm{a}, 1}$, \\ A. Lee Swindlehurst ${ }^{b}$ \\ ${ }^{a}$ Department of Signal Theory and Communications, Universitat Politècnica de \\ Catalunya. C/Jordi Girona, 1-3, Campus Nord UPC, D5. 08034 Barcelona, \\ SPAIN \\ ${ }^{\mathrm{b}}$ Department of Electrical and Computer Engineering, Brigham Young University. \\ Provo, UT 84602, USA.
}

\begin{abstract}
The synchronization of a desired user transmitting a known training sequence in a direct-sequence (DS) asynchronous code-division multiple-access (CDMA) system is addressed. It is assumed that the receiver consists of an arbitrary antenna array and works in a near-far, frequency-nonselective, slowly fading channel. The estimator that we propose is derived by applying the maximum likelihood (ML) principle to a signal model in which the contribution of all the interfering components (e.g., multiple-access interference, external interference and noise) is modeled as a Gaussian term with an unknown and arbitrary space-time correlation matrix. The main contribution of this paper is the fact that the estimator makes efficient use of the structure of the signals in both the space and time domains. Its performance is compared with the Cramér-Rao Bound, and with the performance of other methods proposed recently that also employ an antenna array but only exploit the structure of the signals in one of the two domains, while using the other simply as a means of path diversity. It is shown that the use of the temporal and spatial structures is necessary to achieve synchronization in heavily loaded systems or in the presence of directional external interference.
\end{abstract}

Key words: Synchronization; CDMA; Antenna Arrays; Maximum Likelihood Estimation; Space-Time Diversity.

1 Corresponding author: Tel.: +34 934016431; fax: +34 934016447

2 E-mail: gonzalo@gps.tsc.upc.es, juan@gps.tsc.upc.es, swindle@ee.byu.edu

3 Work supported in part by the Catalan and Spanish Governments under grants: CIRIT 2000SGR-00083, TIC98-0703, TIC99-0849, TIC2000-1025, FIT-0700002000-649, and by the US National Science Foundation under grant CCR-99072.

Preprint submitted to Elsevier Preprint 9th February 2001 


\section{INTRODUCTION}

Multiple-access interference (MAI) is inherent to asynchronous DS-CDMA systems, since orthogonality among the users' codes cannot in general be achieved. The MAI can make the conventional detector (i.e., a bank of filters, each matched to a specific user's code) become useless when the powers of the signals received from different users are unequal [1]. This is the so-called near-far problem. One alternative to overcome this problem is the use of power-control schemes. However, these schemes have some limitations because they increase the overall complexity of the system, do not guarantee optimal performance (e.g., they limit the performance of users with good channels, and some MAI still occurs even though ideal power control is used), and there are certain system configurations in which power control cannot be employed. Therefore, in many communications systems the use of multi-user detectors (usually, in combination with power control) is necessary in order to combat the near-far problem. The optimum multi-user receiver proposed in [2] has been followed by a number of sub-optimum ones (see [3] for a review). All these receivers require knowledge of one or several parameters, such as the users' code timings, powers and carrier phases. Moreover, in general the code timing ${ }^{4}$ needs to be estimated with high accuracy, since timing errors have a large impact on the performance of many detectors [4]. For these reasons, the use of near-far resistant and accurate code synchronization techniques for acquisition and tracking is essential to achieve good performance in a DS-CDMA system. This statement is corroborated by the suggestion in [5] that the capacity of a DS-CDMA system is limited by the ability to achieve code acquisition. Besides, MAI is not the only type of interference that may be encountered. The receiver can be disturbed by any other intentional or accidental signal, which we will represent in general as external interference. The design of synchronization techniques that are also robust against external interference is of fundamental importance in many situations, such as in military or safety-critical applications.

The conventional approaches to timing acquisition and tracking are the sliding correlator and the delay lock loop (DLL) [6]. These schemes are only well suited for an additive white Gaussian noise channel. Extensions of the DLL which are appropriate for a frequency-selective channel were developed in $[7,8]$. However, these modified loops are not able to combat MAI. Recently, several near-far resistant timing estimators have been proposed in the literature for a single-antenna receiver $[9,10,11,12,13,14,15,16]$. Some of these are derived from the maximum likelihood principle and need training sequences. Others exploit the eigenstructure of the correlation matrix of the received signals. While these

$\overline{4}$ We will use the expressions code timing, time delay or code synchronization without distinction, since all of them have been widely employed in the literature. 
estimators do not require training sequences, their performance is poorer than that of the ML-based ones. In [11], a large sample ML estimator was proposed, and a comparison with many other methods revealed that the ML estimator is preferred for moderate or large training sequence lengths. Its accuracy can be significantly improved for short training sequences using a structured estimate of the correlation matrix, as suggested in [13]. This approximate ML estimator has been extended to the case of frequency-selective channels in [15]. However, the resulting criterion involves a complex multidimensional search, so iterative optimization algorithms are considered.

It is well known that detection performance in DS-CDMA can be greatly improved through the use of antenna arrays $[17,18]$. Similarly, the synchronization problem can also benefit from using multiple antennas, as shown in $[19,20,21,22,23]$. Moreover, given the possible lack of temporal structure of the external interference, the use of an antenna array is almost mandatory to achieve robustness against this type of interference. In this paper, we propose a method for estimating the timing of a certain user that transmits a known training sequence. We will focus on code synchronization because there exist a multitude of algorithms for estimating the remaining parameters given reliable estimates of the code-timings [24]. In fact, the expression of the ML estimates of the amplitudes and phases of the signals will be obtained as a by-product of the derivation of the timing estimator in Section 4.1. We assume that the receiver consists of an arbitrary antenna array that operates in a frequency-nonselective (or flat), slowly fading channel [1]. Actually, the estimator proposed herein could also be used in frequency-selective channels, but we will restrict ourselves to the nonselective case for the sake of simplicity. Flat-fading channels are common in situations where the distance between the users and the base station is relatively small (e.g., in a microcell), or when the multipath is due to local scatterers near the remote user or the base station. Note that the availability of a training sequence is not a stringent assumption, since most communications systems transmit these sequences during certain intervals. Besides, once a reliable estimate of the timing is formed, the estimator can be switched to a decision-directed mode. The fact that the method estimates the parameters of only one user while retaining near-far resistance is also of interest, because it leads to decentralized implementations and dramatically reduces the complexity with respect to methods that estimate the parameters of all users simultaneously (see, e.g., [10]).

Following an approach that has already been applied successfully to this and other problems, all signals except that of the desired user are modeled together as a Gaussian component with arbitrary and unknown correlation matrix. This idea has been used for the problem at hand in [11,13,19,21,22] among others, for Doppler and direction-of-arrival estimation in radar systems in [25], and for time-delay estimation in navigation systems in [26]. An extension of [11] for a multiple-sensor receiver can be found in [20]. However, this approach assumes 
that the interfering signals are uncorrelated among antennas, and reduces to several single-sensor estimators applied in parallel to several independent channels. Hence the effect of the antenna array is only to increase the signalto-noise ratio (SNR) and provide diversity to combat the fading of the signal of the desired user at different antennas (i.e., maximal ratio combining); the array is not used to cancel the interfering signals relying on their directional properties. Indeed, as pointed out by the authors themselves, the performance of the estimator in [20] cannot be significantly improved by increasing the number of antennas, when for fair comparisons with single-antenna methods, the interference power is proportional to the number of sensors used in the receiver. In a slowly fading environment, the assumption of uncorrelation among antennas is not appropriate at all, because the signals possess definite spatial signatures, as will be justified in the next section. This fact is exploited by the estimator proposed in [19]. Nevertheless, this estimator assumes that the interfering signals are white in the temporal domain, so only the spatial structure of the MAI is used to combat it. As a result, a prohibitively large number of antennas may be needed to achieve near-far resistance.

The significance of this paper lies in that we consider a space-time correlation matrix for the interfering signals, which allows both the temporal (provided by the codes) and spatial (provided by the antenna array) structure of the received signals to be exploited. The benefits in symbol detection of exploiting the joint space-time signature have been analyzed thoroughly in [27]. Notwithstanding, the use of the space-time signature in synchronization is an open issue. The method proposed herein extends and outperforms those presented in previous work. It will be shown that the use of the spatial and temporal structure of the interference is indispensable in achieving code synchronization in some scenarios, and this can be accomplished with a small number of antennas. The technique in [22] also takes into account the spatial and temporal structure of the interference. But it considers a frequency-selective channel and is limited to estimating the overall channel response, since the estimation of the time delays is computationally too complex.

This paper is organized as follows. In Section 2, the signal model is introduced. Section 3 justifies the essential assumption on which the estimator relies and compares it with the assumptions made in related work. The derivation of the ML estimator and some alternatives to improve the estimate of the correlation matrix are presented in Section 4. Section 5 is concerned with the CramérRao Bound for the problem at hand. Finally, numerical results are analyzed in Section 6, and Section 7 summarizes our conclusions. 


\section{SIGNAL MODEL}

Consider an asynchronous DS-CDMA system with $K$ users and an arbitrary receive antenna array of $L$ sensors, which satisfies the standard narrow-band array condition common to many array signal processing problems (i.e., the time required for a signal to propagate across the array is much smaller than the reciprocal of its bandwidth). We assume a flat-fading channel, which means that for each user the time-delay differences between different propagation paths are negligible compared with the reciprocal of the signal bandwidth [1]. For this channel, the received complex baseband signal at the $l$ th sensor is after down-conversion and chip-matched filtering (see Figure 1)

$$
y_{l}(t)=\sum_{k=1}^{K} \alpha_{l, k} s_{k}\left(t-\tau_{k}\right)+n_{l}(t) \quad l=1,2, \ldots, L
$$

where $\alpha_{l, k}$ is the complex fading coefficient for the $k$ th user at the $l$ th antenna, $\tau_{k}$ is the delay associated with the $k$ th user, and $n_{l}(t)$ represents the thermal noise and all other external interferences. Note that the signal model does not presume the use of power control. The expression in (1) and the development below would also be valid for the received signal before chip-matched filtering. However, we have not considered this case for consistency with the existing literature, and because dealing with the signals after filtering allows us to work with rectangular transmitted chip-pulses in a natural way [10].

The term in (1) corresponding to the $k$ th user's signal is:

$$
s_{k}(t)=\sum_{m=-1}^{M-1} d_{k}(m) c_{k}(t-m T)
$$

where

$$
c_{k}(t)=\sum_{n=0}^{N-1} g_{k}(n) p\left(t-n T_{c}\right)
$$

is the spreading waveform. We have assumed that the summation in (2) starts at $m=-1$ only for the sake of notational convenience in subsequent definitions. The symbols $d_{k}(m)$ are transmitted at a rate $1 / T$ and constitute an i.i.d. sequence with variance $\sigma_{d}^{2}$. The length of the chip sequence $g_{k}(n)$ is $N=T / T_{c}$, the chip rate is $1 / T_{c}$ and $p(t)$ represents an arbitrary chipshaping waveform. The signal is observed during an interval of $M+1$ symbols $\left(T_{o b s}=(M+1) T\right)$, which is the length of the training sequence.

The coefficients $\alpha_{l, k}$ include the effects of the propagation, transmitted power, carrier phase and Doppler frequency. Their temporal evolution is characterized by the coherence time $T_{c o h}$, which is defined as the time interval during which 
a given fading coefficient is highly correlated with itself, and is in general inversely proportional to the maximum Doppler frequency [1]. Since we consider a slowly fading channel (i.e., $T_{c o h} \gg T_{o b s}$ ), for the estimator derivation we will assume that the fading coefficients are constant during the observation interval, as done in many other papers (e.g., $[21,15])$. This assumption is primarily for mathematical convenience, and it will be shown that the performance of the proposed estimator is also highly satisfactory in more realistic scenarios. The condition for slow fading imposes some restrictions on the length of the training sequence $M$ and on the maximum allowable Doppler frequency. However, these are mild restrictions, which are satisfied by the parameters in a large majority of practical situations and do not represent a significant limitation of our approach, as shown in the numerical examples of Section 7. The relevant implication of having fairly constant fading coefficients during $T_{o b s}$ is that the signals possess rather definite spatial signatures, which can be used to differentiate the desired user's signal from the MAI and external interference. It is important to remark that this property holds independently of the statistical correlation between the fading coefficients at different antennas.

The signals in (1) are sampled at the rate $1 / T_{s}=Q / T_{c}$, where $Q$ is an integer and is referred to as the oversampling factor. Each set of $N Q$ consecutive samples received at the $l$ th antenna is stacked into a column vector ${ }^{5}$ :

$$
\mathbf{y}_{l}(m)=\left[y_{l}\left(m T+T_{s}\right) \ldots y_{l}\left(m T+N Q T_{s}\right)\right]^{T}
$$

The sampling is assumed to be completely asynchronous, the only condition being that bit synchronization of the desired user has been previously achieved, i.e., $\tau_{1} \in[0, T)$, where without loss of generality we have assumed that the first user is the desired one. If the duration of the transmitted chip-shaping waveform is $T_{c}$ or smaller, only two consecutive symbols from the desired user contribute to $\mathbf{y}_{l}(m)$. For instance, this occurs with rectangular chip-shaping pulses and is a good approximation for other pulse types. In any case, if the adjacent bits are also present in that vector due to the tails of the chip-shaping pulse, then their tiny contribution will be lumped together in the noise term, as justified in [28]. Therefore, the signal contribution of the first (desired) user to the vector $\mathbf{y}_{l}(m)$ can be expressed as follows

$$
\mathbf{y}_{l, 1}(m)=\alpha_{l, 1} \mathbf{A}^{(1)}\left(\tau_{1}\right) \mathbf{d}_{1}(m)
$$

5 The transpose, conjugate and conjugate transpose operations are represented by $(\cdot)^{T},(\cdot)^{c}$ and $(\cdot)^{H}$, respectively. The $i$ th element of a vector is represented by $[\cdot]_{i}$. 
where

$$
\begin{aligned}
\mathbf{d}_{1}(m) & =\left[d_{1}(m) d_{1}(m-1)\right]^{T} \\
\mathbf{A}^{(1)}\left(\tau_{1}\right) & =\left[\mathbf{a}_{+}^{(1)}\left(\tau_{1}\right) \mathbf{a}_{-}^{(1)}\left(\tau_{1}\right)\right] \\
{\left[\mathbf{a}_{+}^{(1)}\left(\tau_{1}\right)\right]_{i} } & =c_{1}\left(i T_{s}-\tau_{1}\right) \quad i=1, \ldots, N Q \\
{\left[\mathbf{a}_{-}^{(1)}\left(\tau_{1}\right)\right]_{i} } & =c_{1}\left(i T_{s}+T-\tau_{1}\right) \quad i=1, \ldots, N Q .
\end{aligned}
$$

The matrix $\mathbf{A}^{(1)}\left(\tau_{1}\right)$ contains the temporal signatures of the desired user. Note that the model above is also valid for users other than the first one as well. To simplify our notation, in the sequel we will drop the the superscript that indexes the users. At this point, we can write the received $N Q \times 1$ vector at the $l$ th sensor as

$$
\mathbf{y}_{l}(m)=\alpha_{l, 1} \mathbf{A}\left(\tau_{1}\right) \mathbf{d}_{1}(m)+\mathbf{e}_{l}(m) \quad m=0,1, \ldots, M-1
$$

The vector $\mathbf{e}_{l}(m)$ includes the MAI, the thermal noise and all other sources of interference.

\section{GAUSSIAN ASSUMPTION}

If the temporal vectors received from every antenna are stacked into a $L N Q \times 1$ space-time vector:

$$
\mathbf{y}(m)=\left[\mathbf{y}_{1}^{T}(m) \mathbf{y}_{2}^{T}(m) \ldots \mathbf{y}_{L}^{T}(m)\right]^{T},
$$

then equation (10) can be rewritten in a compact form as

$$
\mathbf{y}(m)=\left(\boldsymbol{\alpha} \otimes \mathbf{A}\left(\tau_{1}\right)\right) \mathbf{d}_{1}(m)+\mathbf{e}(m),
$$

where $\otimes$ denotes the Kronecker product, e $(m)$ is formed similarly to $\mathbf{y}(m)$ and

$$
\boldsymbol{\alpha}=\left[\begin{array}{llll}
\alpha_{1,1} & \alpha_{2,1} & \ldots & \alpha_{L, 1}
\end{array}\right]^{T}
$$

is the spatial signature of the first user. As outlined in the introduction, we model e $(m)$ as a zero-mean, circularly complex Gaussian $L N Q \times 1$ vector, which is independent of $\mathbf{d}_{1}(m)$ and independent for different samples, and has an arbitrary and unknown space-time covariance matrix:

$$
\mathrm{E}\{\mathbf{e}(m)\}=\mathbf{0} \quad \mathrm{E}\left\{\mathbf{e}(m) \mathbf{e}(n)^{H}\right\}=\mathbf{Q} \delta_{m, n} .
$$


There is no doubt that this model is only approximate. Nevertheless, it gathers the most significant effects of all the interfering signals, and allows us to derive tractable algorithms. The problem addressed in this paper may be stated as follows. Estimate $\tau_{1}$, given the set of samples

$$
\mathbf{Y}=[\mathbf{y}(0) \mathbf{y}(1) \cdots \mathbf{y}(M-1)]
$$

and assuming that the spreading sequence $\left\{g_{1}(n)\right\}_{n=0}^{N-1}$ and the training bit sequence $\left\{d_{1}(m)\right\}_{m=-1}^{M-1}$ for the desired user are available. Estimates of $\boldsymbol{\alpha}$ and $\mathrm{Q}$, which are taken as deterministic and unstructured parameters, will also be derived. Although we do not parameterize the spatial signature in terms of one or several directions-of-arrival and amplitudes, the array maintains its ability to discriminate the signals in the spatial domain. Assuming an unstructured $\boldsymbol{\alpha}$ eliminates the need for a calibrated antenna array, and allows us to model a cluster of coherent arrivals that share the same time delay, without estimating the individual parameters of each arrival. A detailed discussion of the advantages of this assumption can be found in [23].

It is well known that the assumption of temporally white MAI is unrealistic [29] and leads to non near-far resistant estimators because it neglects the structure of the MAI. However, the estimator proposed herein, though modeling the interference as Gaussian, does not suffer from the same problem since it retains the structure of the MAI in the matrix $\mathbf{Q}$, and so it is near-far resistant. Actually, it is the fact that an unknown correlation matrix $\mathbf{Q}$ is considered for the equivalent noise $\mathbf{e}(\mathrm{m})$ that makes the estimator able to attenuate any interfering signal that exhibits a certain structure in the temporal and/or spatial domains. In this paper, we present the estimator that results from an arbitrary matrix $\mathbf{Q}$, in contrast to previous work that has solved the problem stated herein for simplified structures of that matrix. We will also see in Section 4.2 that imposing a very simple and natural structure on $\mathbf{Q}$ allows us to estimate it without using excessively large training sequences.

The signal model proposed in [19] may seem at first glance rather different to the one proposed above. Nonetheless, it can be shown that they are related by realizing that in [19] it is implicitly assumed that the space-time correlation matrix can be decomposed as $\mathbf{Q}=\mathbf{Q}_{s p} \otimes \mathbf{I}_{N Q}$, where $\mathbf{Q}_{s p}$ is an arbitrary $L \times L$ matrix that corresponds to the spatial correlation of the interference. It is apparent that the estimator in [19] yields suboptimal performance since it ignores the inherent temporal structure of the CDMA signals. A dual decomposition is considered in [20]. In this case, the matrix $\mathbf{Q}$ is expressed as $\mathbf{Q}=\mathbf{I}_{L} \otimes \mathbf{Q}_{t e}$, where $\mathbf{Q}_{t e}$ is a $N Q \times N Q$ matrix representing the temporal structure of the interference. This model amounts to presuming that the fading coefficients $\alpha_{l, k}$ for a given user are uncorrelated between different antennas and that the observation interval is long enough (compared to $T_{c o h}$ ) to apply ergodicity, but this last condition is not satisfied in the case of slow fading. 


\section{MAXIMUM LIKELIHOOD ESTIMATOR}

In this section, the estimator of the code-timing of the desired user is derived by applying the ML principle [30] to the signal model described above. Next, several techniques that may serve to improve the quality of the estimate of the noise-plus-interference correlation matrix are discussed.

\subsection{Derivation}

The probability density function of $\mathbf{Y}$ is

$$
\begin{aligned}
& p\left(\mathbf{Y} \mid \tau_{1}, \boldsymbol{\alpha}, \mathbf{Q}\right)=\frac{1}{L N Q M|\mathbf{Q}|^{M}} \\
& \cdot \exp \left\{-\sum_{m=0}^{M-1}\left(\mathbf{y}(m)-\mathbf{D} \mathbf{d}_{1}(m)\right)^{H} \mathbf{Q}^{-1}\left(\mathbf{y}(m)-\mathbf{D} \mathbf{d}_{1}(m)\right)\right\},
\end{aligned}
$$

where $|\cdot|$ denotes the determinant, and the matrix $\mathbf{D}\left(\tau_{1}, \boldsymbol{\alpha}\right)=\boldsymbol{\alpha} \otimes \mathbf{A}\left(\tau_{1}\right)$ is the joint space-time signature of the desired user. The dependence of $\mathbf{D}$ on the parameters is omitted from our notation whenever there is no risk of confusion. The negative $\log$-likelihood function ${ }^{6}$ of the observed data $\mathbf{Y}$ is easily shown to be

$$
\Lambda_{1}\left(\tau_{1}, \boldsymbol{\alpha}, \mathbf{Q}\right)=\ln |\mathbf{Q}|+\operatorname{Tr}\left\{\mathbf{Q}^{-1} \mathbf{C}\left(\tau_{1}, \boldsymbol{\alpha}\right)\right\},
$$

where $\operatorname{Tr}\{\cdot\}$ denotes the trace of a matrix, and

$$
\mathbf{C}\left(\tau_{1}, \boldsymbol{\alpha}\right)=\frac{1}{M} \sum_{m=0}^{M-1}\left(\mathbf{y}(m)-\mathbf{D} \mathbf{d}_{1}(m)\right)\left(\mathbf{y}(m)-\mathbf{D} \mathbf{d}_{1}(m)\right)^{H} .
$$

The ML estimates of $\tau_{1}, \boldsymbol{\alpha}$ and $\mathbf{Q}$ are the values that minimize (17). Using standard matrix calculus results [31] and without imposing any structure on $\mathbf{Q}$, it is immediate that the gradient of (17) with respect to $\mathbf{Q}$ is

$$
\frac{\partial \Lambda_{1}\left(\tau_{1}, \boldsymbol{\alpha}, \mathbf{Q}\right)}{\partial \mathbf{Q}}=\mathbf{Q}^{-1}-\mathbf{Q}^{-1} \mathbf{C}\left(\tau_{1}, \boldsymbol{\alpha}\right) \mathbf{Q}^{-1}
$$

The value of $\mathbf{Q}$ that nulls (19) is

$$
\hat{\mathbf{Q}}\left(\tau_{1}, \boldsymbol{\alpha}\right)=\mathbf{C}\left(\tau_{1}, \boldsymbol{\alpha}\right) .
$$

provided that $\mathbf{C}\left(\tau_{1}, \boldsymbol{\alpha}\right)$ is non-singular (refer to Section 4.2 for conditions on the minimum value of $M)$. The $\mathrm{ML}$ estimate of $\mathbf{Q}$, denoted by $\hat{\mathbf{Q}}_{M L}$, is

$\overline{6}$ In the sequel, all parameter-independent additive or multiplicative constants of the likelihood functions will be neglected. 
obtained by evaluating (20) at the ML estimates of $\tau_{1}$ and $\boldsymbol{\alpha}$, which are yet to be determined.

Define the following matrices

$$
\begin{aligned}
\hat{\mathbf{D}} & =\left[\hat{\mathbf{d}}_{+} \hat{\mathbf{d}}_{-}\right]=\hat{\mathbf{R}}_{y d} \hat{\mathbf{R}}_{d d}^{-1} & \\
\hat{\mathbf{R}}_{y y} & =\frac{1}{M} \sum_{m=0}^{M-1} \mathbf{y}(m) \mathbf{y}^{H}(m) & \hat{\mathbf{R}}_{d d}=\frac{1}{M} \sum_{m=0}^{M-1} \mathbf{d}_{1}(m) \mathbf{d}_{1}^{H}(m) \\
\hat{\mathbf{R}}_{y d} & =\frac{1}{M} \sum_{m=0}^{M-1} \mathbf{y}(m) \mathbf{d}_{1}^{H}(m) & \hat{\mathbf{W}}=\hat{\mathbf{R}}_{y y}-\hat{\mathbf{R}}_{y d} \hat{\mathbf{R}}_{d d}^{-1} \hat{\mathbf{R}}_{y d}^{H} .
\end{aligned}
$$

Note that the matrices $\hat{\mathbf{D}}$ and $\hat{\mathbf{W}}$ are the unstructured estimates of $\mathbf{D}$ and the noise correlation matrix Q, respectively. When (20) is substituted into (17), the following concentrated likelihood function results

$$
\begin{aligned}
\Lambda_{2}\left(\tau_{1}, \boldsymbol{\alpha}\right) & =\ln \left|\hat{\mathbf{R}}_{y y}-\mathbf{D} \hat{\mathbf{R}}_{y d}^{H}-\hat{\mathbf{R}}_{y d} \mathbf{D}^{H}+\mathbf{D} \hat{\mathbf{R}}_{d d} \mathbf{D}^{H}\right| \\
& =\ln \left|\hat{\mathbf{W}}+(\mathbf{D}-\hat{\mathbf{D}}) \hat{\mathbf{R}}_{d d}(\mathbf{D}-\hat{\mathbf{D}})^{H}\right| \\
& =\ln |\hat{\mathbf{W}}|+\ln \left|\mathbf{I}+\hat{\mathbf{W}}^{-1}(\mathbf{D}-\hat{\mathbf{D}}) \hat{\mathbf{R}}_{d d}(\mathbf{D}-\hat{\mathbf{D}})^{H}\right| \\
& =\ln |\hat{\mathbf{W}}|+\ln \left|\mathbf{I}+(\mathbf{D}-\hat{\mathbf{D}})^{H} \hat{\mathbf{W}}^{-1}(\mathbf{D}-\hat{\mathbf{D}}) \hat{\mathbf{R}}_{d d}\right| .
\end{aligned}
$$

Equation (25) is obtained from (24) by adding and subtracting the term $\hat{\mathbf{R}}_{y d} \hat{\mathbf{R}}_{d d}^{-1} \hat{\mathbf{R}}_{y d}^{H}$ inside the determinant. The other two equations stem directly from the following standard properties of the determinant: $|\mathbf{P} \mathbf{Q}|=|\mathbf{P}| \cdot|\mathbf{Q}|$ and $|\mathbf{I}+\mathbf{P} \mathbf{Q}|=|\mathbf{I}+\mathbf{Q} \mathbf{P}|$, valid for matrices of appropriate dimensions. The function in (27) can be minimized in closed-form with respect to $\boldsymbol{\alpha}$. However, while the derivation is not complicated, it is very cumbersome and will not be presented herein. Next, this estimate of $\boldsymbol{\alpha}$ could be substituted back into (27), and a one-dimensional criterion for the estimation of the delay would result. Instead of minimizing $\Lambda_{2}$, we will transform this function into another that is asymptotically (in $M$, throughout the paper) equivalent and allows a simpler derivation of the estimates. It can be argued that since $M$ is the length of the training sequence, we will never reach asymptotics in $M$. However, our numerical results show that asymptotic behaviour is obtained for rather modest sample sizes.

According to the Weak Law of Large Numbers:

$$
\begin{aligned}
\hat{\mathbf{D}} & =\mathbf{D}\left(\tilde{\tau_{1}}, \tilde{\boldsymbol{\alpha}}\right)+O_{p}(1 / \sqrt{M}) \\
\hat{\mathbf{R}}_{d d} & =\sigma_{d}^{2} \mathbf{I}+O_{p}(1 / \sqrt{M})
\end{aligned}
$$

where $O_{p}(\cdot)$ represents the "in probability" version of the corresponding deterministic notation [32], and $\tilde{\tau}_{1}$ and $\tilde{\boldsymbol{\alpha}}$ are the true values of the parameters. 
Due to equation (28), we can replace $\hat{\mathbf{R}}_{d d}$ by its asymptotic value and neglect the second- and higher-order terms in the Taylor expansion of the second logarithm in (27), while retaining the same asymptotic accuracy for the estimates. The Taylor expansion of the logarithm of the determinant is

$$
\ln |\mathbf{I}+\mathbf{X}|=\operatorname{Tr}\{\mathbf{X}\}-\frac{1}{2} \operatorname{Tr}\left\{\mathbf{X}^{2}\right\}+\cdots
$$

which is valid whenever the absolute values of the eigenvalues of $\mathbf{X}$ are bounded above by one. Therefore, an asymptotically equivalent ML criterion can be expressed as follows

$$
\begin{aligned}
\Lambda_{3}\left(\tau_{1}, \boldsymbol{\alpha}\right) & =\operatorname{Tr}\left\{\left(\mathbf{D}\left(\tau_{1}, \boldsymbol{\alpha}\right)-\hat{\mathbf{D}}\right)^{H} \hat{\mathbf{W}}^{-1}\left(\mathbf{D}\left(\tau_{1}, \boldsymbol{\alpha}\right)-\hat{\mathbf{D}}\right)\right\} \\
& =\left(\boldsymbol{\alpha} \otimes \mathbf{a}_{+}\left(\tau_{1}\right)-\hat{\mathbf{d}}_{+}\right)^{H} \hat{\mathbf{W}}^{-1}\left(\boldsymbol{\alpha} \otimes \mathbf{a}_{+}\left(\tau_{1}\right)-\hat{\mathbf{d}}_{+}\right) \\
& +\left(\boldsymbol{\alpha} \otimes \mathbf{a}_{-}\left(\tau_{1}\right)-\hat{\mathbf{d}}_{-}\right)^{H} \hat{\mathbf{W}}^{-1}\left(\boldsymbol{\alpha} \otimes \mathbf{a}_{-}\left(\tau_{1}\right)-\hat{\mathbf{d}}_{-}\right) .
\end{aligned}
$$

It is convenient to write this cost function in such a way that the linear and quadratic dependences on $\boldsymbol{\alpha}$ are made more explicit, since this facilitates the minimization with respect to this vector. An equivalent expression for (32) is

$$
\begin{aligned}
\Lambda_{3}\left(\tau_{1}, \boldsymbol{\alpha}\right)= & -2 \operatorname{Re}\left\{\boldsymbol{\alpha}^{H} \mathbf{p}_{+}\left(\tau_{1}\right)\right\}+\boldsymbol{\alpha}^{H} \mathbf{F}_{+}\left(\tau_{1}\right) \boldsymbol{\alpha} \\
& -2 \operatorname{Re}\left\{\boldsymbol{\alpha}^{H} \mathbf{p}_{-}\left(\tau_{1}\right)\right\}+\boldsymbol{\alpha}^{H} \mathbf{F}_{-}\left(\tau_{1}\right) \boldsymbol{\alpha} \\
& +\hat{\mathbf{d}}_{+}^{H} \hat{\mathbf{W}}^{-1} \hat{\mathbf{d}}_{+}+\hat{\mathbf{d}}_{-}^{H} \hat{\mathbf{W}}^{-1} \hat{\mathbf{d}}_{-}
\end{aligned}
$$

where we have defined

$$
\begin{aligned}
& \mathbf{p}_{ \pm}\left(\tau_{1}\right)=\operatorname{mat}_{N Q \times L}^{T}\left\{\hat{\mathbf{W}}^{-1} \hat{\mathbf{d}}_{ \pm}\right\} \mathbf{a}_{ \pm}^{c}\left(\tau_{1}\right) \\
& \mathbf{F}_{ \pm}\left(\tau_{1}\right)=\left(\mathbf{I}_{L} \otimes \mathbf{a}_{ \pm}^{H}\left(\tau_{1}\right)\right) \hat{\mathbf{W}}^{-1}\left(\mathbf{I}_{L} \otimes \mathbf{a}_{ \pm}\left(\tau_{1}\right)\right),
\end{aligned}
$$

and the $\operatorname{mat}_{N Q \times L}\{\cdot\}$ operator rearranges column-wise the elements of one vector into a $N Q \times L$ matrix. At this point, the minimization of (33) with respect to $\boldsymbol{\alpha}$ is immediate and yields

$$
\hat{\boldsymbol{\alpha}}_{M L}=\left.\left(\mathbf{F}_{+}\left(\tau_{1}\right)+\mathbf{F}_{-}\left(\tau_{1}\right)\right)^{-1}\left(\mathbf{p}_{+}\left(\tau_{1}\right)+\mathbf{p}_{-}\left(\tau_{1}\right)\right)\right|_{\tau_{1}=\hat{\tau}_{1, M L}} .
$$

After substituting (36) into (33), the timing estimator is obtained as

$$
\begin{aligned}
\hat{\tau}_{1, M L}=\arg \max _{\tau_{1}}\left(\mathbf{p}_{+}\left(\tau_{1}\right)+\mathbf{p}_{-}\left(\tau_{1}\right)\right)^{H} \\
\cdot\left(\mathbf{F}_{+}\left(\tau_{1}\right)+\mathbf{F}_{-}\left(\tau_{1}\right)\right)^{-1}\left(\mathbf{p}_{+}\left(\tau_{1}\right)+\mathbf{p}_{-}\left(\tau_{1}\right)\right),
\end{aligned}
$$

which only involves the minimization of a one-dimensional cost function. 
A possible way to extend this estimator to frequency-selective channels consists in modifying the signal model in (12). The new model should explicitly take into account the fact that the desired user's signal arrives at the antenna array through $R_{1}$ propagation paths having different delays and different spatial signatures. This is the approach followed in [15] for a single-antenna receiver. Its serious drawback is that the application of the ML principle to the new signal model results in a highly complex multidimensional optimization problem. However, it is important to remark that a much simpler alternative is also possible. It simply consists of employing the cost function derived for flat-fading channels, given by (37), also for frequency-selective channels. The estimates of the delays of the $R_{1}$ propagation paths are obtained as the values corresponding to the largest $R_{1}$ maxima of that cost function, while in the flat-fading case only the absolute maximum is picked. This simple modification is possible since the delay-spacing between the different propagation paths is generally greater than the reciprocal of the signal bandwidth (see [33]). A similar approach was used in [33] to extend a MUSIC-based estimator, initially derived for flat-fading channels, to the frequency-selective case. In any case, our paper is focused on the flat-fading case, and a deeper analysis for other types of channels is beyond the scope of our effort.

\subsection{Covariance Matrix Estimation}

The advantage of exploiting the space-time structure of the signals is that every antenna adds $N Q$ degrees of freedom to the system, whereas each user occupies only two degrees and each external interferer occupies between 1 and $N Q$ depending on its bandwidth. When the channel introduces angular and Doppler spread, the number of degrees of freedom used by each signal increases. Using spatio-temporal diversity, a large number of degrees of freedom is achieved with few sensors. The price to be paid is that a longer training sequence may be needed, at least theoretically, with respect to other approaches that only exploit one source of diversity but provide a much smaller number of degrees of freedom. In order that $\hat{\mathbf{W}}$ be non-singular with probability one, we need $M \geq L N Q+2$, which may result in too large of a training sequence. To shed light on how this restriction on $M$ can be alleviated, it is convenient to analyze the eigenstructure of the correlation matrix $\mathbf{Q}$. The eigendecomposition of $\mathbf{Q}$ is given by

$$
\mathbf{Q}=\mathbf{V} \Sigma \mathbf{V}^{H}
$$

where $\boldsymbol{\Sigma}$ is a diagonal matrix of the eigenvalues $\left\{\lambda_{i}\right\}$ of $\mathbf{Q}$ in descending order, and the columns of $\mathbf{V}$ are the corresponding eigenvectors. The eigenvalues satisfy the following relation

$$
\lambda_{1} \geq \cdots \geq \lambda_{d} \geq \lambda_{d+1}=\cdots=\lambda_{L N Q}=\sigma_{w}^{2},
$$


where $d$ is the dimension of the interference subspace, which is spanned by the first $d$ columns of $\mathbf{V}$, and $\sigma_{w}^{2}$ is the power of the white noise. The subspace spanned by the last $L N Q-d$ columns of $\mathbf{V}$ is referred to as the noise subspace.

Since $\hat{\mathbf{W}}$ is a consistent estimate of $\mathbf{Q}$, the eigenvalues of $\hat{\mathbf{W}}$ tend to those of $\mathrm{Q}$ when $M$ grows without limit. However, if $M<L N Q+2$, some eigenvalues of $\hat{\mathbf{W}}$ are zero, and hence the inverse of this matrix does not exist. Moreover, when $M$ is only slightly greater that $L N Q+2$, some eigenvalues of $\hat{\mathbf{W}}$ may be very small. The inversion of an ill-conditioned matrix can cause numerical instability and impair the performance of the estimator. Nevertheless, the inequality $M>L N Q+2$ should not be a necessary condition for the application of the estimator, since as long as $M$ is much greater than $d$, appropriate estimates of the interference and noise subspaces of $\mathbf{Q}$ can be obtained. To this end, a parametric estimate of $\mathbf{Q}$ is derived, which we designate $\hat{\mathbf{W}}_{s}$. This new estimate is obtained from $\hat{\mathbf{W}}$, but we force it to have the structure given by (38) and (39), instead of being fully unstructured. The matrix $\hat{\mathbf{W}}_{s}$ is taken as the one that is closest to $\hat{\mathbf{W}}$ in the sense of the Frobenius-norm. Thus, assuming that $\hat{\mathbf{W}}_{s}=\hat{\mathbf{P}}+\hat{\sigma}^{2} \mathbf{I}$ and that the rank of $\hat{\mathbf{P}}$ is $d$, the problem that we have to solve can be stated as

$$
\left[\hat{\mathbf{P}} \hat{\sigma}^{2}\right]=\arg \min _{\sigma^{2}, \mathbf{P} \mid \operatorname{rank}\{\mathbf{P}\}=d}\left\|\hat{\mathbf{W}}-\mathbf{P}-\sigma^{2} \mathbf{I}\right\|_{F}^{2} .
$$

It is well-known that the solution to this problem is given by

$$
\begin{aligned}
\hat{\mathbf{P}} & =\sum_{k=1}^{d}\left(\hat{\lambda}_{k}-\hat{\sigma}^{2}\right) \hat{\mathbf{v}}_{k} \hat{\mathbf{v}}_{k}^{H} \\
\hat{\sigma}^{2} & =\frac{1}{L N Q-d} \sum_{k=d+1}^{L N Q} \hat{\lambda}_{k},
\end{aligned}
$$

where $\hat{\mathbf{v}}_{k}$ and $\hat{\lambda}_{k}$ are the eigenvectors and eigenvalues of $\hat{\mathbf{W}}$. The use of the structured estimate $\hat{\mathbf{W}}_{s}$ in (36) and (37) not only avoids the previous bound on $M$ for the application of the estimator, but also improves the performance for all values of $M$. We assume that an estimate of the dimension $d$ is available. It can be inferred either from knowledge of certain system parameters, such as the number of active users, angular spread, etc., or by applying multiplicity tests on the smallest eigenvalues of $\hat{\mathbf{W}}[34,35,36]$. This is a non-trivial issue that is beyond the scope of the paper. The same approach was used to obtain a structured estimate of the correlation matrix in [13,15], but the 2-norm was used instead. In [37] a related method, known as "eigenvalue thresholding", is proposed and derived using the ML principle with a noise floor constraint. It involves an eigendecomposition and requires knowledge of the white noise power, but not knowledge of the rank of the interference subspace.

An alternative technique for eliminating the problems associated with inverting $\hat{\mathbf{W}}$ is to use the widespread diagonal loading technique [38]. It simply 
consists in replacing $\hat{\mathbf{W}}$ by another estimate $\hat{\mathbf{W}}_{d}$ obtained as

$$
\hat{\mathbf{W}}_{d}=\hat{\mathbf{W}}+\lambda \mathbf{I}
$$

where $\lambda$ should be on the order of $\sigma_{w}^{2}$. This is an ad hoc technique whose advantage lies in its simplicity. However, simulation results have shown that the previous eigenanalysis method performs slightly better. In [13], it is suggested that this improvement is due to the fact that the noise subspace of $\hat{\mathbf{W}}_{s}$ is white (i.e., flat eigenvalue spectrum), whereas the noise subspaces of $\hat{\mathbf{W}}$ and $\hat{\mathbf{W}}_{d}$ are colored.

An approach that may seem logical at first glance is to replace the inverse of $\hat{\mathbf{W}}$, when this matrix is singular, by its Moore-Penrose pseudo-inverse $\hat{\mathbf{W}}^{\#}$. However, the use of the pseudo-inverse is not recommended because it yields a notably worse performance than the two previous approaches, and its calculation is computationally complex. The explanation for its poor performance is simple. In order to mitigate the effect of the interference, the terms $\hat{\mathbf{d}}_{ \pm}$and $\mathbf{I}_{L} \otimes \mathbf{a}_{ \pm}\left(\tau_{1}\right)$ are prewhitened with the inverse of the noise-plus-interference correlation matrix, as shown in (34)-(35). The dominant component after prewhitening should be the projection of these terms onto the noise subspace, since it is orthogonal to the interference ${ }^{7}$. Whenever the matrix $\hat{\mathbf{W}}$ is non-singular, this desired property is achieved by any of the approaches above (that is, using $\hat{\mathbf{W}}^{-1}=\hat{\mathbf{W}}^{\#}, \hat{\mathbf{W}}_{s}^{-1}$ or $\hat{\mathbf{W}}_{d}^{-1}$ ), since the eigenvalues of the noise subspace are much smaller than the rest. However, when any of the noise eigenvalues are zero, the pseudo-inverse disregards the projection onto the corresponding eigenvectors. On the contrary, the eigenanalysis method and the diagonal loading approach assign small values to the null eigenvalues, so that the projection onto the corresponding eigenvectors is emphasized.

The discussion above does not pretend to be a formal or complete study of the application of the ML principle with singular or ill-conditioned correlation matrices. This is an open issue that deserves a much deeper analysis. Our goal has only been to show that the unstructured estimate of the correlation matrix can be suitably transformed in order to deal with short training sequences.

\section{CRAMER-RAO BOUND}

The Cramér-Rao Bound (CRB) is a lower bound on the covariance of any unbiased estimator [30]. It can be proved that the ML estimators (36), (20) and (37) are consistent as long as the signals to which they are applied satisfy

\footnotetext{
7 In this discussion we are implicitly assuming that the interference is much more powerful than the background noise. This situation is common in mobile communication systems, which are usually interference-limited.
} 
the model presented in Sections 2 and 3. The consistency of $\hat{\tau}_{1, M L}$ and $\hat{\boldsymbol{\alpha}}_{M L}$ follows immediately from equations (28) and (32); and along with equation (20) it directly implies the consistency of $\hat{\mathbf{Q}}_{M L}$. The proofs of these claims are well-known (see, e.g., [39]). Since all the ML estimators are consistent, they are also asymptotically efficient [40, Theorem 6.2.3], that is, their asymptotic covariance coincides with the CRB.

According to the model under consideration, the $M$ observations of $\mathbf{y}(m)$ are independent circular Gaussian vectors with mean $\boldsymbol{\mu}(m)=\mathbf{D}\left(\tau_{1}, \boldsymbol{\alpha}\right) \mathbf{d}_{1}(m)$ and covariance $\mathbf{Q}$. The parameters of this model are $\boldsymbol{\eta}=\left[\operatorname{Re}\left\{\boldsymbol{\alpha}^{T}\right\}, \operatorname{Im}\left\{\boldsymbol{\alpha}^{T}\right\}, \tau_{1}\right]^{T}$ and $\mathbf{Q}^{8}$. The Bangs-Slepian's formula [41, ch. 15] for the klth element of the Fisher Information Matrix (FIM) is

$$
[\mathbf{F I M}]_{k l}=M \operatorname{Tr}\left\{\mathbf{Q}^{-1} \mathbf{Q}_{k}^{\prime} \mathbf{Q}^{-1} \mathbf{Q}_{l}^{\prime}\right\}+2 \operatorname{Re}\left\{\sum_{m=0}^{M-1}\left(\boldsymbol{\mu}_{k}^{\prime}(m)\right)^{H} \mathbf{Q}^{-1} \boldsymbol{\mu}_{l}^{\prime}(m)\right\}
$$

where $(\cdot)_{k}^{\prime}$ denotes the derivative with respect to the $k$ th parameter. Since $\boldsymbol{\mu}(m)$ and $\mathbf{Q}$ depend on different parameters, the FIM is block diagonal with respect to $\boldsymbol{\eta}$ and $\mathbf{Q}$. Therefore, the CRB for $\boldsymbol{\eta}$ is the same whether $\mathbf{Q}$ is known or not, and vice versa. As we are concerned only with the CRB for the signal parameters $(\boldsymbol{\eta})$, we need only consider the second term in (44). The CRB for $\boldsymbol{\eta}$ is the inverse of the corresponding block of the FIM, and can be written as

$$
\mathbf{C R B}^{-1}(\boldsymbol{\eta})=2 \operatorname{Re}\left\{\left[\begin{array}{ccc}
\mathbf{F}_{1} & j \mathbf{F}_{1} & \mathbf{F}_{2} \boldsymbol{\alpha} \\
-j \mathbf{F}_{1}^{H} & \mathbf{F}_{1} & -j \mathbf{F}_{2} \boldsymbol{\alpha} \\
\boldsymbol{\alpha}^{H} \mathbf{F}_{2}^{H} & j \boldsymbol{\alpha}^{H} \mathbf{F}_{2}^{H} & \boldsymbol{\alpha}^{H} \mathbf{F}_{3} \boldsymbol{\alpha}
\end{array}\right]\right\}
$$

where

$$
\begin{aligned}
\mathbf{F}_{1} & =\sum_{m=0}^{M-1}\left(\mathbf{I}_{L} \otimes\left(\mathbf{A}\left(\tau_{1}\right) \mathbf{d}_{1}(m)\right)^{H}\right) \mathbf{Q}^{-1}\left(\mathbf{I}_{L} \otimes\left(\mathbf{A}\left(\tau_{1}\right) \mathbf{d}_{1}(m)\right)\right) \\
\mathbf{F}_{2} & =\sum_{m=0}^{M-1}\left(\mathbf{I}_{L} \otimes\left(\mathbf{A}\left(\tau_{1}\right) \mathbf{d}_{1}(m)\right)^{H}\right) \mathbf{Q}^{-1}\left(\mathbf{I}_{L} \otimes\left(\mathbf{B}\left(\tau_{1}\right) \mathbf{d}_{1}(m)\right)\right) \\
\mathbf{F}_{3} & =\sum_{m=0}^{M-1}\left(\mathbf{I}_{L} \otimes\left(\mathbf{B}\left(\tau_{1}\right) \mathbf{d}_{1}(m)\right)^{H}\right) \mathbf{Q}^{-1}\left(\mathbf{I}_{L} \otimes\left(\mathbf{B}\left(\tau_{1}\right) \mathbf{d}_{1}(m)\right)\right) \\
\mathbf{B}\left(\tau_{1}\right) & =\left[\mathbf{b}_{+}\left(\tau_{1}\right) \mathbf{b}_{-}\left(\tau_{1}\right)\right]=\frac{\mathrm{d} \mathbf{A}\left(\tau_{1}\right)}{\mathrm{d} \tau_{1}} .
\end{aligned}
$$

8 Actually, the parameters corresponding to $\mathbf{Q}$ are its real and imaginary parts. For sake of simplicity, this fact is not detailed since it does not affect the computation of the CRB for $\boldsymbol{\eta}$. 
It is possible to compute the asymptotic values of (46)-(48). Recalling that $\hat{\mathbf{R}}_{d d} \rightarrow \sigma_{d}^{2} \mathbf{I}$, it is not hard to verify that

$$
\begin{aligned}
& \mathbf{F}_{1}^{a}=M \lim _{M \rightarrow \infty} \frac{\mathbf{F}_{1}}{M}=M \sigma_{d}^{2}\left(\left(\mathbf{I}_{L} \otimes \mathbf{a}_{+}^{H}\left(\tau_{1}\right)\right) \mathbf{Q}^{-1}\left(\mathbf{I}_{L} \otimes \mathbf{a}_{+}\left(\tau_{1}\right)\right)+\right. \\
& \left.+\left(\mathbf{I}_{L} \otimes \mathbf{a}_{-}^{H}\left(\tau_{1}\right)\right) \mathbf{Q}^{-1}\left(\mathbf{I}_{L} \otimes \mathbf{a}_{-}\left(\tau_{1}\right)\right)\right) \\
& \mathbf{F}_{2}^{a}=M \lim _{M \rightarrow \infty} \frac{\mathbf{F}_{2}}{M}=M \sigma_{d}^{2}\left(\left(\mathbf{I}_{L} \otimes \mathbf{a}_{+}^{H}\left(\tau_{1}\right)\right) \mathbf{Q}^{-1}\left(\mathbf{I}_{L} \otimes \mathbf{b}_{+}\left(\tau_{1}\right)\right)+\right. \\
& \left.+\left(\mathbf{I}_{L} \otimes \mathbf{a}_{-}^{H}\left(\tau_{1}\right)\right) \mathbf{Q}^{-1}\left(\mathbf{I}_{L} \otimes \mathbf{b}_{-}\left(\tau_{1}\right)\right)\right) \\
& \mathbf{F}_{3}^{a}=M \lim _{M \rightarrow \infty} \frac{\mathbf{F}_{3}}{M}=M \sigma_{d}^{2}\left(\left(\mathbf{I}_{L} \otimes \mathbf{b}_{+}^{H}\left(\tau_{1}\right)\right) \mathbf{Q}^{-1}\left(\mathbf{I}_{L} \otimes \mathbf{b}_{+}\left(\tau_{1}\right)\right)+\right. \\
& \left.+\left(\mathbf{I}_{L} \otimes \mathbf{b}_{-}^{H}\left(\tau_{1}\right)\right) \mathbf{Q}^{-1}\left(\mathbf{I}_{L} \otimes \mathbf{b}_{-}\left(\tau_{1}\right)\right)\right) \text {. }
\end{aligned}
$$

When these values are substituted into (45), an asymptotic expression for the CRB is obtained. The asymptotic CRB is usually preferred because it does not depend on the particular value of the training sequence, and is the one plotted in the figures of the next section.

\section{SIMULATION RESULTS}

In this section we compare the performance of our estimator, referred to as the "space-time diversity" (STD) estimator, with two of the techniques proposed to date that in most cases give the best results. Namely, we consider the methods presented in [19] and [20], which we will denote as "space-diversity" (SD) and "time-diversity" (TD) estimators, respectively. In these two papers and also in [11], the SD and TD methods are compared with a number of different estimators proposed in the literature. Note that the comparison with these two approaches is fair since they also use an antenna array in reception. The computational complexity of the SD and TD methods is smaller than that of the STD estimator, because they work with the space-only and the timeonly correlation matrices of the interfering signals, respectively. The size of these two matrices is obviously smaller than that of the space-time correlation matrix employed in the the STD approach. Here, we analyze two performance measures:

- Probability of acquisition $\left(P_{a c}\right)$. We define a correct acquisition to have occurred when the delay estimate is within a half-chip of the true value, i.e., $\left|\hat{\tau}_{1, M L}-\tilde{\tau}_{1}\right|<T_{c} / 2$. 
- The root mean squared error (RMSE) given correct acquisition, i.e.,

$$
\operatorname{RMSE}\left(\tau_{1}\right)=\sqrt{\mathrm{E}\left\{\left|\hat{\tau}_{1, M L}-\tilde{\tau}_{1}\right|^{2}|| \hat{\tau}_{1, M L}-\tilde{\tau}_{1} \mid<T_{c} / 2\right\}}
$$

This measure is relevant for the tracking operation of the estimators.

A method is considered to have failed when $P_{a c} \leq 0.5$, due to the large number of outliers. The RMSE is not plotted in this case. We have observed that the three estimators under consideration are essentially unbiased (their biases are much smaller than their standard deviations). Therefore, the RMSEs are for all practical purposes identical to the standard deviations. All results are obtained from 1000 Monte Carlo realizations. The simulation conditions, except when one of them is varied, are as follows:

- (pseudo-)Gold codes with length $N=15$ chips and BPSK modulation.

- rectangular chip-shaped pulses and oversampling factor $Q=1$.

- Energy per bit to white-noise spectral density ratio (Eb/No) equal to $4 \mathrm{~dB}$ per antenna for the desired user.

- uniform linear array with $L=4$ antennas spaced 0.5 wavelengths apart.

- $K=10$ users, $M=80$ training bits.

- The power of the signal from each interfering user is distributed log-normally with mean 10dB (with respect to the desired user) and standard deviation $10 \mathrm{~dB}$. This distribution models the log-normal fading caused by largedistance reflectors.

- The delays of the users, the mean directions-of-arrival (DOA) of the users and the external interference are randomly chosen from the range of all possible values and fixed throughout the Monte Carlo realizations.

The near-far ratio (NFR) is defined as the ratio between the mean power of each interfering user and that of the desired user. The structured estimate of the correlation matrix $\hat{\mathbf{W}}_{s}$ is used in the implementation of the STD estimator. We simulate two different channels. The first, referred to as the static channel, is a channel that remains constant during the observation interval. This corresponds to the situation that we have considered in the signal model of Section 2. There is no angular spreading, so each user has a unique spatial signature. Since the Doppler frequency $f_{d}$ is assumed equal to 0 , the signals do not suffer from multiplicative distortion (or fast-fading), only log-normal fading. The amplitude and phase of each user's signal are held fixed during the observation interval, but are varied for each Monte Carlo run in order to model the log-normal fading.

The second channel is a realistic mobile channel for the uplink. It is generated according to the spatio-temporal model described in [42]. Each signal arrives at the array through several rays, all of them with the same delay. The number of rays follows a truncated Poisson law with mean and maximum 
values equal to 25 and 50 rays, respectively. The directions of arrival of the rays are generated according to a Gaussian distribution with a given mean and a standard deviation of 5 degrees. This is the value that characterizes the angular spread of the signals. The total power of each signal is divided among its propagation rays following a Laplacian law conditioned on the separation between the DOA of each ray and the mean DOA of the signal (see [42] for details). The Doppler spectrum has the classical Clarke's bath-shape [1], obtained by assuming multiple and randomly located reflectors near the mobile, with normalized maximum Doppler frequency equal to $f_{d} T=2 \cdot 10^{-3}$. Therefore, the multiplicative distortion introduced by the channel has approximately a correlation duration of $1 / f_{d} T=500$ symbols. This value for the factor $f_{d} T$ corresponds to a system with an typical set of parameters, such as $900 \mathrm{MHz}$ carrier frequency, $50 \mathrm{~kb} / \mathrm{s}$ data rate and $120 \mathrm{~km} / \mathrm{h}$ speed, or $1800 \mathrm{MHz}$ carrier frequency, $100 \mathrm{~kb} / \mathrm{s}$ data rate and $120 \mathrm{~km} / \mathrm{h}$ speed. Note that for a pedestrian channel the speed is about $3 \mathrm{~km} / \mathrm{h}$, and therefore the value of $f_{d} T$ is much smaller.

We first consider the effect of the length of the training sequence $M$. The results are shown in Figure 2. The estimator proposed in this paper is the only one that attains the CRB for the static channel, even though the Gaussian assumption is only an approximate one. This fact corroborates the explanation in Section 3 stating that the Gaussian model with space-time correlation is reasonable and models the most significant effects of the MAI. The CRB is achieved for lengths of the training sequence larger than 250 bits. For smaller values, there is a very slight degradation with respect to the CRB, which causes the difference between the RMSE and the CRB present in all the subsequent figures. As expected, the performance of all the estimators deteriorates in the mobile channel, where the RMSE can not be further reduced by increasing $M$. This impairment should not be interpreted as a failure of the estimators, but only as the effect of working in a much more adverse environment, and it will be visible in all the following results. As $M$ increases, the multiplicative distortion blurs the signal of the desired user. Then, the effective length of the training sequence is no longer equal to $M$, but is bounded by the temporal correlation of the channel. Also in the mobile channel the STD estimator outperforms the other two approaches. The SD method possesses the largest RMSE and the lowest $P_{a c}$, since it is the approach with the smallest number of degrees of freedom. Figure 2(b) demonstrates the ability of our algorithm to acquire the desired user's delay. As shown in Figure 2(a), RMS errors between 0.1 and 0.01 chips can be achieved with windows of less than 100 bits, indicating that the algorithm can be used for tracking slowly time-varying parameters in decision-directed mode.

In Figure 3, we investigate the effect of varying the number of users. This has special interest for a base station that uses spatial-division multiple-access (SDMA), since such systems may have more users than the length of the codes. 
Again the STD estimator gives better results than the other two methods both in RMSE and $P_{a c}$. The SD and TD approaches experience a serious deterioration, specially in their probabilities of acquisition, when the number of users exceeds the length of the code (i.e., $K>N$ ), and they completely fail when $K>2 N$ in the scenario under consideration. On the other hand, using the space-time estimator the number of users may be increased beyond twice the code length without an excessive degradation. For instance, note that for a static channel with $K=40$ users the probability of acquisition remains virtually equal to 1 , and only goes down to 0.82 for the mobile channel.

Next, the effect of a wide-band external interference is analyzed in Figure 4. Because of its large bandwidth, the interference does not show any temporal structure, so it can be exclusively mitigated in the spatial domain. Only the STD and SD estimators provide adequate performance when the desired signal-to-interference ratio (SIR) is small (e.g., smaller than $<-15 \mathrm{~dB}$ ). Their performance is nearly insensitive to the SIR except for extremely low SIR in the mobile channel. Despite everything, the former gives better results than the latter in all cases. Moreover, the SD estimator is not near-far resistant for the system parameters that we have considered. In Figure 5 the near-far resistance of the different estimators is compared. In the static channel, the CRB and the RMSE and $P_{a c}$ of the estimator proposed herein are totally insensitive to the MAI level, whereas those of the TD and SD schemes are not. With regard to the probability of acquisition, the STD estimator performs satisfactorily in the mobile channel up to a NFR equal to $35 \mathrm{~dB}$, which is an improvement of about $8 \mathrm{~dB}$ and $18 \mathrm{~dB}$ over the TD and SD methods, respectively. Further insight into the near-far performance is gained by observing Figure 6. An estimator can be considered to be near-far resistant when the RMSE tends to zero and the $P_{a c}$ tends to one as the Eb/No increases, even in the presence of arbitrarily strong MAI. This property is only satisfied by our STD estimator, at least in the static channel. The RMSEs of the other two estimators (i.e., the SD and TD methods) have performance floors due to the MAI that cannot be surpassed by reducing the power of the background white noise.

In Figure 7, we examine the relationship between the probability of acquisition and the RMSE with the normalized Doppler $f_{d} T$. These results are obtained for an angular spread with standard deviation equal to 8 degrees. The STD estimator performs better than the other two for all values of $f_{d} T$ considered. The difference between the RMSEs of the different methods is roughly constant as the Doppler is increased. On the other hand, the probability of acquisition of the STD estimator is less sensitive to the Doppler than that of the SD and TD approaches. This figure shows that the performance of the estimator proposed herein is not critically affected by the Doppler spread of the channel. For instance, $P_{a c}$ for our method is approximately 0.92 when $f_{d} T=0.01$. This is an excellent result, since the correlation length of the channel is about 100 
symbols, and hence on the order of the observation interval.

Our last set of results involves analyzing the performance achieved with different estimates of the correlation matrix. In Figure 8, we compare the RMSEs obtained with the structured estimate $\hat{\mathbf{W}}_{s}^{-1}$ (the one employed in all the simulations above), the pseudo-inverse $\hat{\mathbf{W}}^{\#}$ and the diagonally loaded estimate $\hat{\mathbf{W}}_{d}^{-1}$. The diagonal loading factor is set equal to the power of the white noise. As predicted and justified by the theoretical study in Section 4.2, the performance with the pseudo-inverse is always worse than with the other two estimates, and undergoes a severe degradation for short lengths of the training sequence. The RMSEs obtained with the diagonally loaded and the structured matrix estimates, which are nearly coincident, are better discerned in Figure 9. This figure shows that the RMSE of the former is noticeably greater than that of the latter for small loading factors. When the loading factor is equal to or greater than the white-noise power, they perform similarly, but there is always a certain advantage in favor of the structured estimate, especially for the mobile channel.

\section{CONCLUSIONS}

A code-timing synchronization technique for DS-CDMA systems that operates in near-far, frequency-nonselective, slowly fading channels and employs an arbitrary antenna array for reception has been derived by applying the ML principle. As such the technique is a single-user, near-far resistant estimator and would be applicable in a system employing multiuser detection without power control. It is assumed for the derivation that the desired user transmits a known training sequence, and all other received components are modeled as Gaussian with unknown space-time correlation. This approach fully exploits the spatial and temporal structure of the interfering signals in order to cancel them, and differs from other methods put forward to date that, while also employing antenna arrays, only exploit the structure of the signals in one of the domains. As a result, the proposed technique outperforms existing synchronization methods for reasonable lengths of the training sequence. The use of a structured estimate of the correlation matrix or diagonal loading allows one to reduce the required size of the observation window. The RMSE and the acquisition probability of the proposed algorithm have been evaluated numerically in two types of channels. Although the estimator is applied in a multiple-access channel, the RMSE attains the CRB derived under the Gaussian assumption, which confirms the validity of the starting model. The results of this paper show that the efficient use of space-time diversity is indispensable for accurate acquisition and tracking of the synchronization parameters in heavily loaded systems and/or in the presence of external interference. 


\section{References}

[1] J. Proakis, Digital Communciations. New York: McGraw-Hill, 3rd ed., 1995.

[2] S. Verdú, "Minimum Probability of Error for Asynchronous Gaussian MultipleAccess Channels," IEEE Trans. on Information Theory, vol. 32, pp. 85-96, Jan. 1986.

[3] S. Verdú, Multiuser Detection. Cambridge University Press, 1998.

[4] S. Parkvall, E. Ström, and B. Ottersten, "The Impact of Timing Errors on the Performance of Linear DS-CDMA Receivers," IEEE Journal on Selected Areas on Communications, vol. 14, pp. 1660-1668, Oct. 1996.

[5] U. Madhow and M. B. Pursley, "Acquisition-Based Capacity of Direct-Sequence Spread-Spectrum Communication Networks," in Proc. of the Conference of Information Sciences and Systems, pp. 1186-1189, March 1991.

[6] J. K. Holmes, Coherent Spread Spectrum Systems. Krieger Press, 1990.

[7] W. H. Sheen and G. L. Stüber, "A New Tracking Loop for Spread-Spectrum Systems on Frequency-Selective Fading Channels," IEEE Trans. on COM, vol. 43, pp. 3063-3072, Dec. 1995.

[8] W. H. Sheen and C. H. Tai, "A Noncoherent Tracking Loop With Diversity and Multipath Interference Cancellation for Direct-Sequence Spread-Spectrum Systems," IEEE Trans. on COM, vol. 46, pp. 1516-1524, Nov. 1998.

[9] R. F. Smith and S. L. Miller, "Code Timing Estimation in a NearFar Environment for Direct-Sequence Code-Division Multiple-Access," in Proc. IEEE Military Conf., pp. 47-51, 1994.

[10] E. Ström, S. Parkvall, S. Miller, and B. Ottersten, "Propagation Delay Estimation in Asynchronous Direct-Sequence Code-Division Multiple Access Systems," IEEE Trans. on COM, vol. 44, pp. 84-93, Jan. 1996.

[11] D. Zheng, J. Li, S. Miller, and E. Ström, "An Efficient Code-Timing Estimator for DS-CDMA Signals," IEEE Trans. SP, vol. 45, pp. 82-89, Jan. 1997.

[12] S. Bensley and B. Aazhang, "Subspace-Based Channel Estimation for CDMA Communication Systems," IEEE Trans. Commun., pp. 1009-1020, Aug. 1996.

[13] S. Bensley and B. Aazhang, "Maximum-Likelihood Synchronization of a Single User for Code-Division Multiple-Access Communication Systems," IEEE Trans. on COM, vol. 46, pp. 392-399, March 1998.

[14] T. Östman, S. Parkvall, and B. Ottersten, "An Improved MUSIC Algorithm for Estimation of Time Delays in Asynchronous DS-CDMA Systems," IEEE Trans. on COM, vol. 47, pp. 1628-1631, Nov. 1999.

[15] E. Ertin, U. Mitra, and S. Siwamogsatham, "Maximum-Likelihood Based Multipath Channel Estimation for Code-Division Multiple-Access Systems." Accepted for publication in IEEE Trans. on COM, 2000. 
[16] J. Romero-García, R. D. Gaudenzi, F. Giannetti, and M. Luise, "A Frequency Error Resistant Blind CDMA Detector," IEEE Trans. COM, vol. 48, pp. 10701076, July 2000.

[17] A. Paulraj and C. Papadias, "Space-Time Processing for Wireless Communications," IEEE Signal Processing Magazine, vol. 14, pp. 49-83, Nov. 1997.

[18] K. Molnar and G. Bottomley, "Adaptive Array Processing MLSE Receivers for TDMA Digital Cellular/PCS Communications," IEEE J. Select. Areas Commun., vol. 16, pp. 1340-1351, Oct. 1998.

[19] Z.-S. Liu, J. Li, and S. Miller, "A Receiver Diversity Based Code-Timing Estimator for Asynchronous DS-CDMA Systems," in Proc. ICASSP, vol. VI, (Seattle, WA), pp. 3245-3248, 1998.

[20] Z.-S. Liu, J. Li, and S. Miller, "An Efficient Code-Timing Estimator for Receiver Diversity DS-CDMA Systems," IEEE Trans. COM, vol. 46, pp. 826-835, June 1998.

[21] A. Jakobsson, A. Swindlehurst, D. Astély, and C. Tidestav, "A Blind Frequency Domain Method for DS-CDMA Synchronization Using Antenna Arrays," in Proc. 32nd Asilomar Conf. on Signals, Systems and Computers, (Pacific Groove, CA), Nov. 1998.

[22] C. Sengupta, J. R. Cavallaro, and B. Aazhang, "Maximum Likelihood Multipath Channel Parameter Estimation in CDMA Systems Using Antenna Arrays," in Proc. PIMRC, 1998.

[23] G. Seco, A. L. Swindlehurst, and D. Astély, "Exploting Antenna Arrays for Synchronization," in Signal Processing Advances in Wireless Communications (G. B. Giannakis, Y. Hua, P. Stoica, and L. Tong, eds.), vol. II: Trends in Singleand Multi-User Systems, ch. 10, Prentice-Hall, 2000.

[24] Z. Xie, C. K. Rushforth, R. T. Short, and T. K. Moon, "Joint Signal Detection and Parameter Estimation in Multiuser Communications," IEEE Trans. on COM, vol. 41, pp. 1208-1215, Aug. 1993.

[25] A. Swindlehurst and P. Stoica, "Maximum Likelihood Methods in Radar Array Signal Processing," Proceedings of the IEEE, vol. 86, pp. 421-441, Feb. 1998.

[26] G. Seco and J. Fernández-Rubio, "Maximum Likelihood Propagation-Delay Estimation in Unknown Correlated Noise using Antenna Arrays: Application to Global Navigation Satellite Systems," in Proc. ICASSP, vol. IV, (Seattle, WA), pp. 2065-2068, May 1998.

[27] D. Astély, Spatial and Spatio-Temporal Processing with Antenna Arrays in Wireless Systems. PhD thesis, Royal Institute of Technology, KTH, Stockholm, Sweden, 1999.

[28] U. Madhow, "Blind Adaptive Interference Suppression for the Near-Far Resistant Acquisition and Demodulation of Direct-Sequence CDMA Signals," IEEE Trans. on SP, vol. 45, pp. 124-136, Jan. 1997. 
[29] S. Verdú, "Demodulation in the Presence of Multiuser Interference: Progress and Misconceptions," in Intelligent Methods in Signal Processing and Communications (D. Docampo, A. Figueiras-Vidal, and F. Pérez-González, eds.), pp. 15-44, Birkhauseer, Boston, MA, 1997.

[30] L. L. Scharf, Statistical Signal Processing, Detection, Estimation, and Time Series Analysis. Addison-Wesley, 1990.

[31] J. R. Magnus and H. Neudecker, Matrix Differential Calculus with Applications in Statistics and Econometrics. John Wiley \& Sons, 1999.

[32] P. J. Brockwell and R. A. Davis, Time Series: Theory and Methods. New York: Springer-Verlag, 2 ed., 1991.

[33] S. Parkvall, Near-Far Resistant DS-CDMA Systems: Parameter Estimation and Data Detection. PhD thesis, Royal Institute of Technology, KTH, Stockholm, Sweden, 1996.

[34] J. Rissanen, "Modeling by Shortest Data Description," Automatica, vol. 14, pp. $465-471,1978$.

[35] H. Akaike, "A New Look at Statistical Model Identification," IEEE Trans. on Automatic Control, vol. 19, pp. 716-723, 1974.

[36] M. Wax and T. Kailath, "Detection of Signals by Information Theoretic Criteria," IEEE Trans. on ASSP, vol. 33, pp. 387-392, April 1985.

[37] K. Harmancı, J. Tabrikian, and J. L. Krolik, "Relationships Between Adaptive Minimum Variance Beamforming and Optimal Source Location," IEEE Trans. on $S P$, vol. 48, pp. 1-12, Jan. 2000.

[38] B. D. Carlson, "Covariance Matrix Estimation Errors and Diagonal Loading in Adaptive Arrays," IEEE Trans. on AES, vol. 24, pp. 397-401, July 1988.

[39] T. Söderström and P. Stoica, System Identification. London, UK: Prentice Hall International, 1989.

[40] E. L. Lehmann, Theory of Point Estimation. New York: Wiley, 1983.

[41] S. M. Kay, Fundamentals of Statistical Signal Processing. Estimation Theory. Prentice Hall, 1993.

[42] K. Pedersen, P. Mogensen, and B. Fleury, "A Stochastic Model of the Temporal and Azimuthal Dispersion Seen at the Base Station in Outdoor Propagation Environments," IEEE Trans. on Vehicular Technology, vol. 49, pp. 437-447, May 2000. 


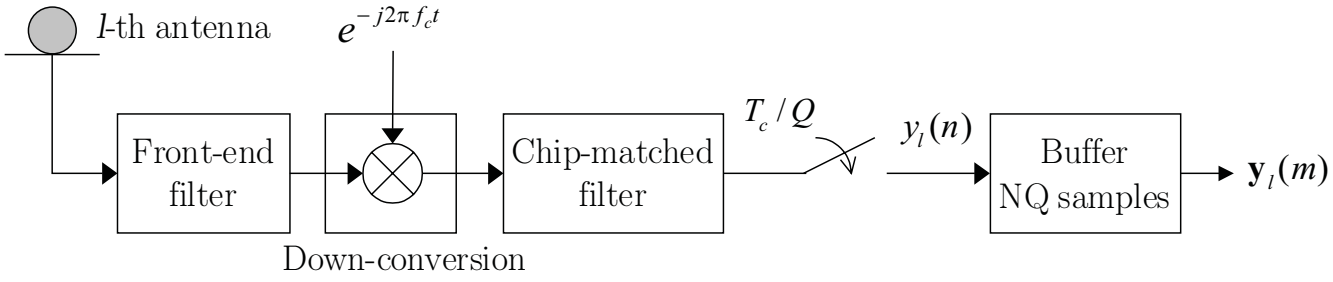

Figure 1. Demodulator block diagram. 


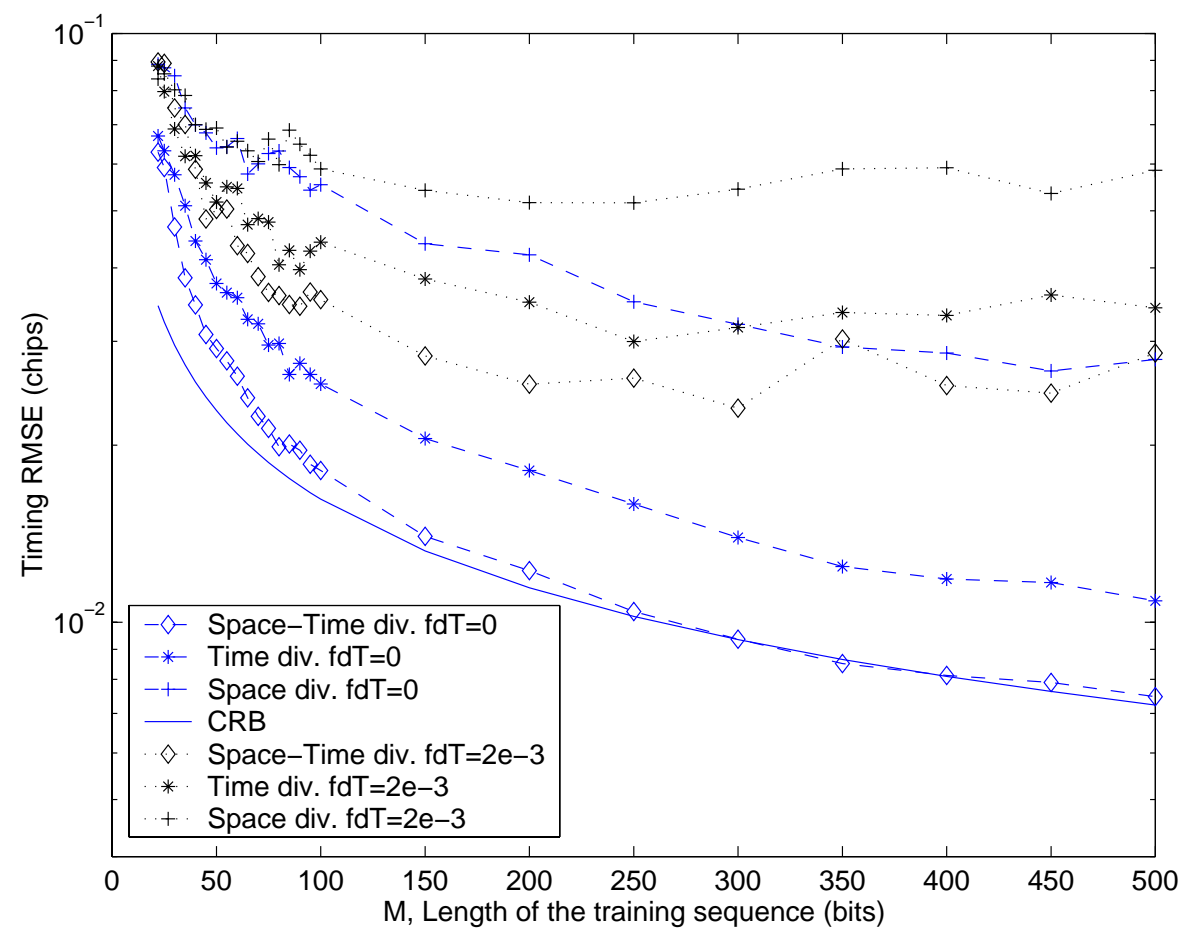

(a) RMSE

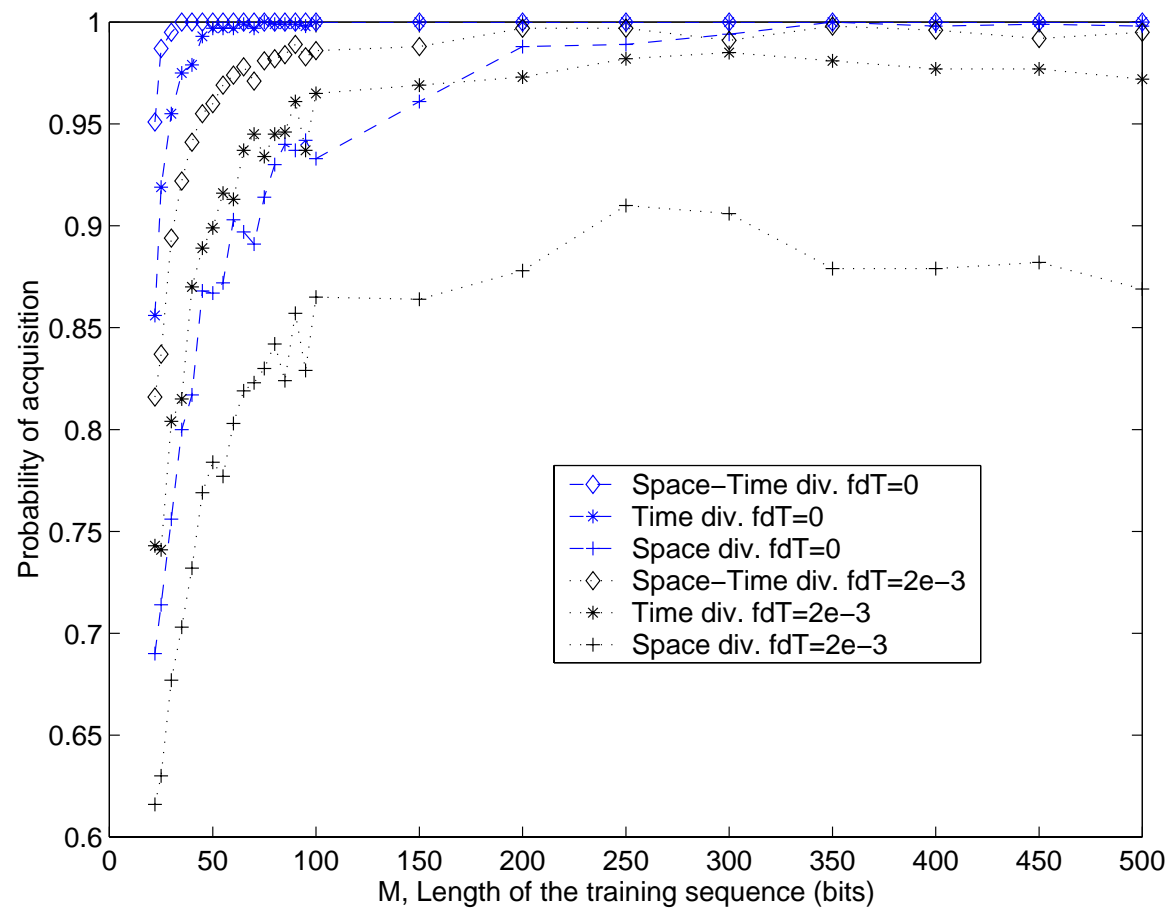

(b) Probability of acquisition

Figure 2. Performance of the STD, TD and SD estimators as a function of the length of the training sequence $M$ in two different channels. $K=10, N=15, L=4, \mathrm{~Eb} / \mathrm{No}=4 \mathrm{~dB}$ per antenna, $\mathrm{NFR}=10 \mathrm{~dB}$. 


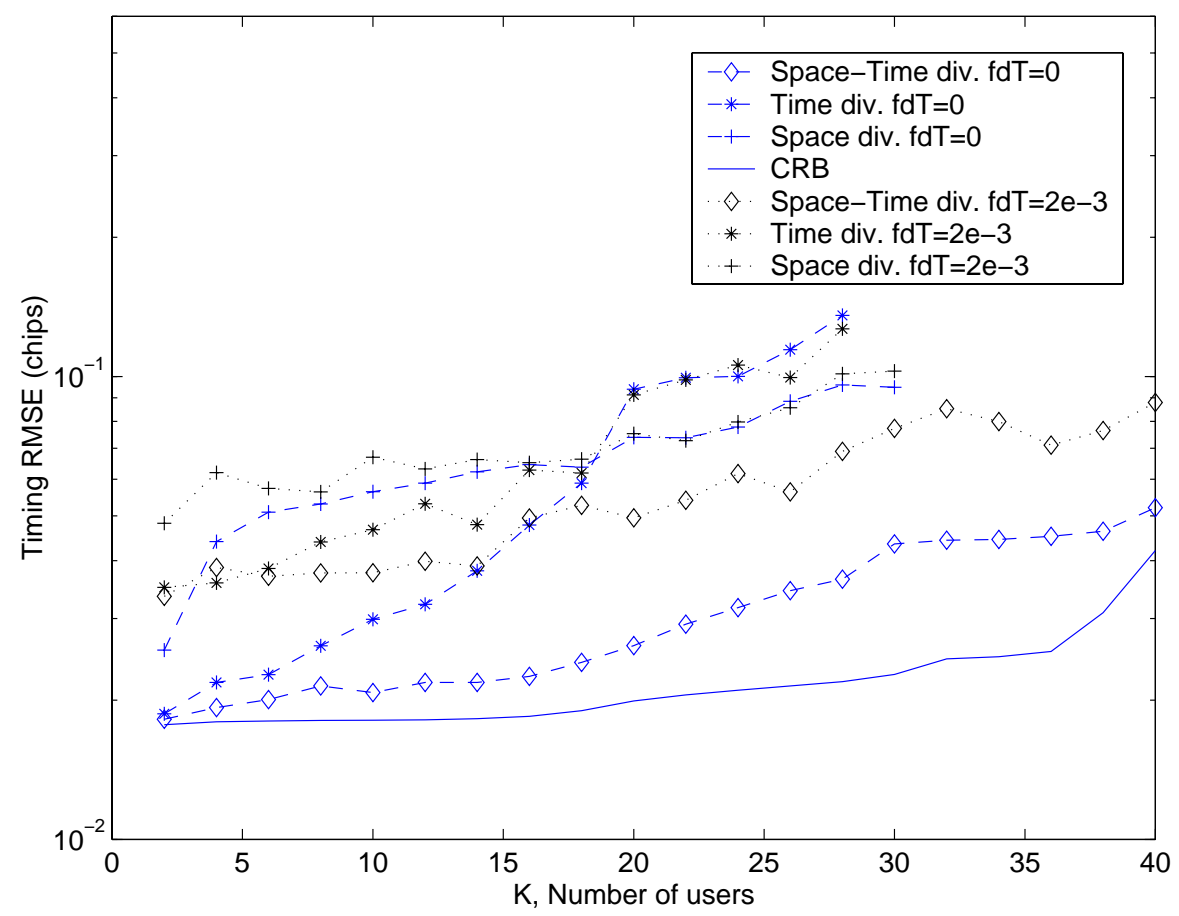

(a) RMSE

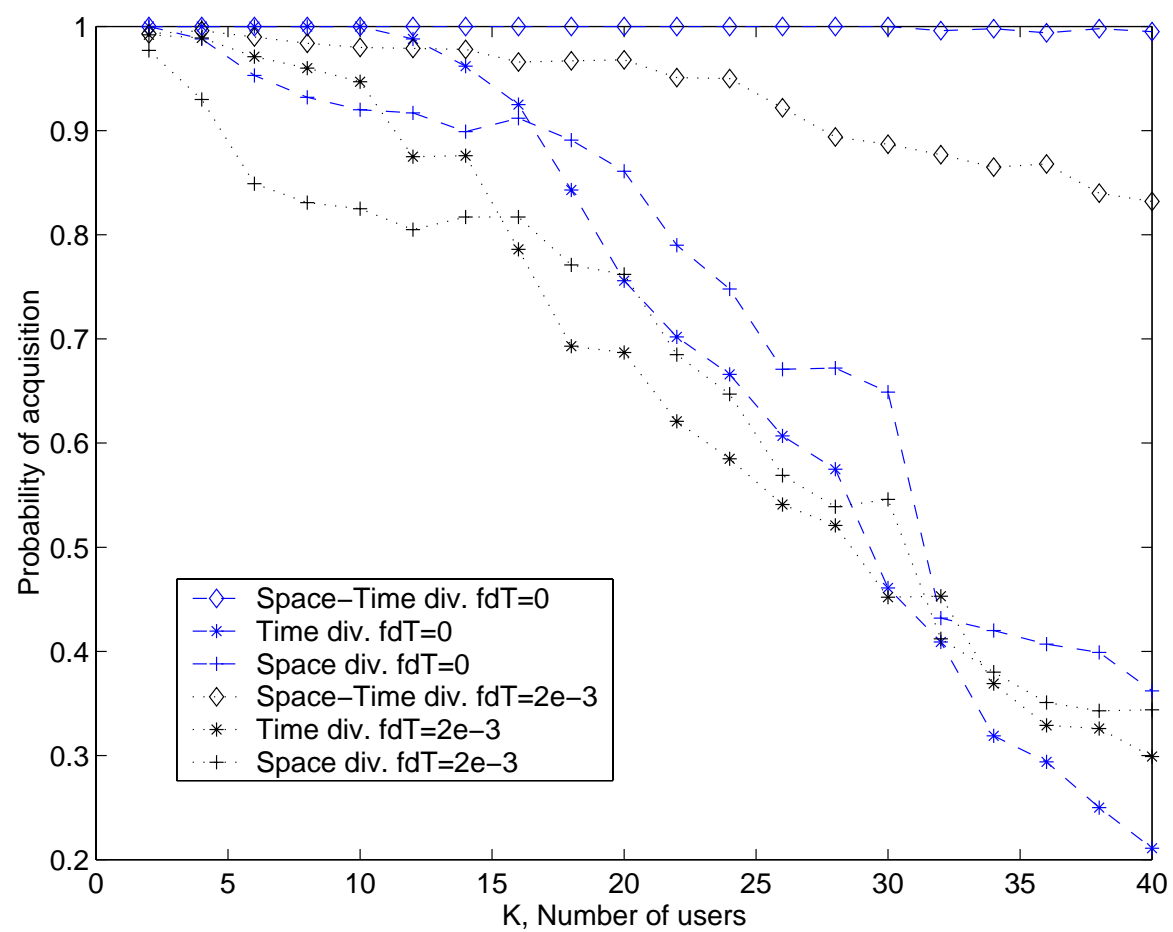

(b) Probability of acquisition

Figure 3. Performance of the STD, TD and SD estimators as a function of the number of users $K$ in two different channels. $M=80, N=15, L=4, \mathrm{~Eb} / \mathrm{No}=4 \mathrm{~dB}$ per antenna, NFR $=10 \mathrm{~dB}$. 


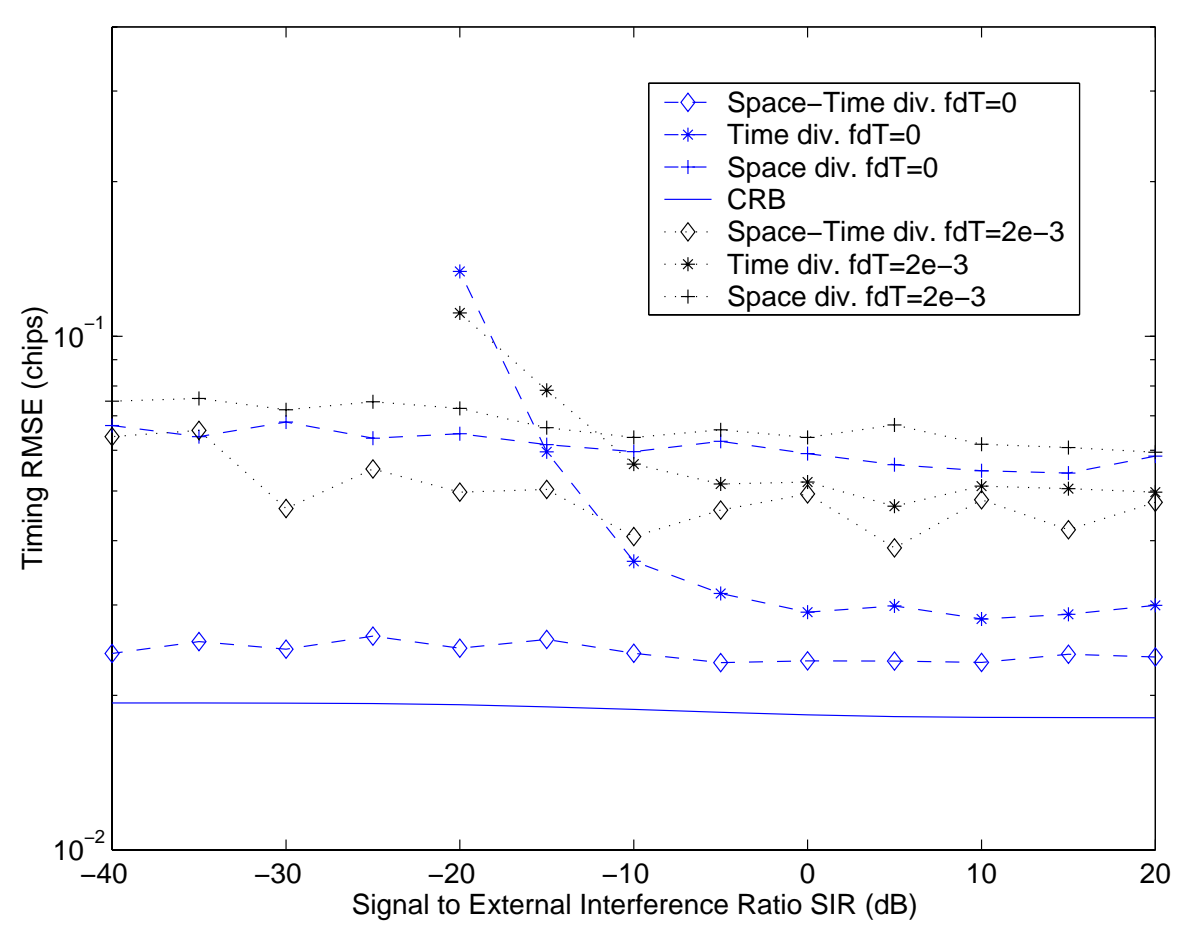

(a) RMSE

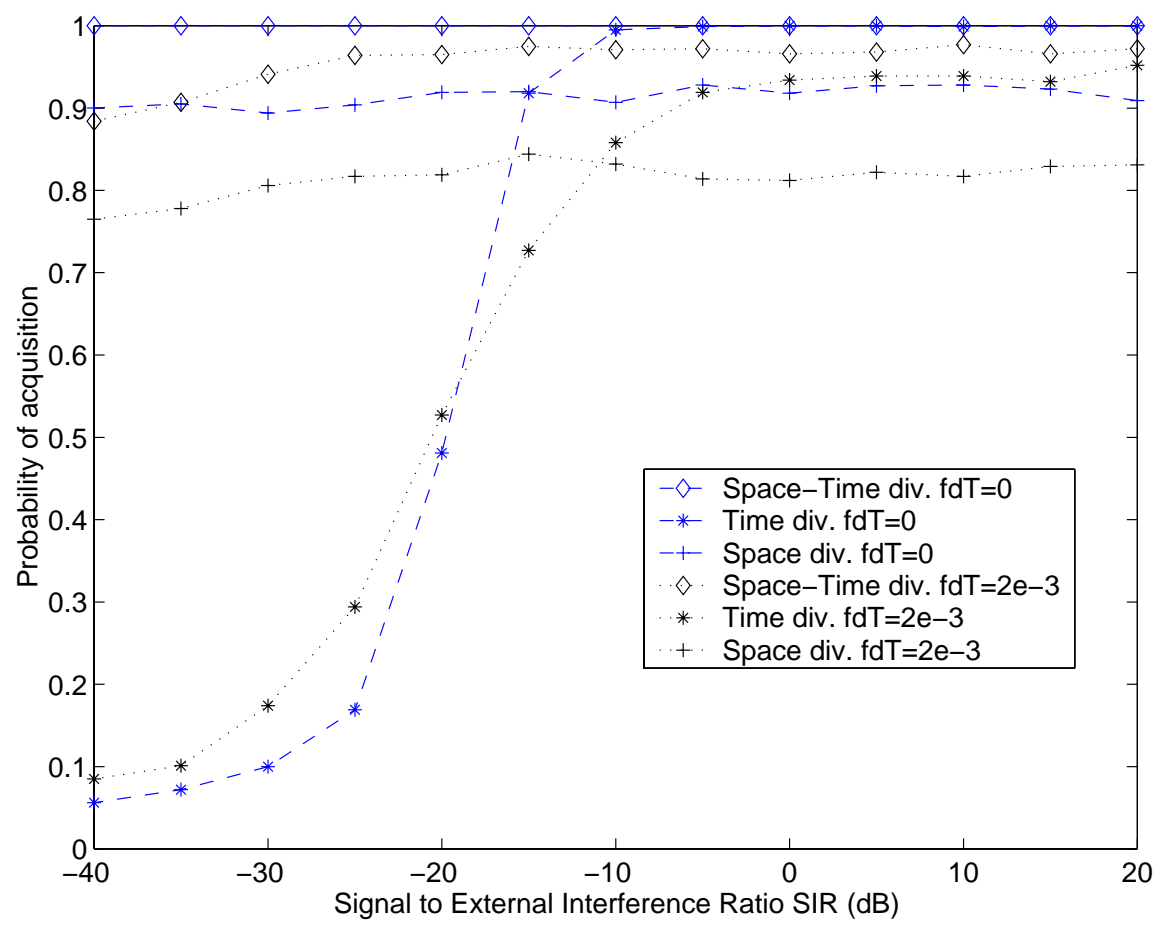

(b) Probability of acquisition

Figure 4. Performance of the STD, TD and SD estimators in the presence of a wide-band external interference in two different channels. $M=80, K=10 ; N=15, L=4, \mathrm{~Eb} / \mathrm{No}=4 \mathrm{~dB}$ per antenna, NFR $=10 \mathrm{~dB}$. 


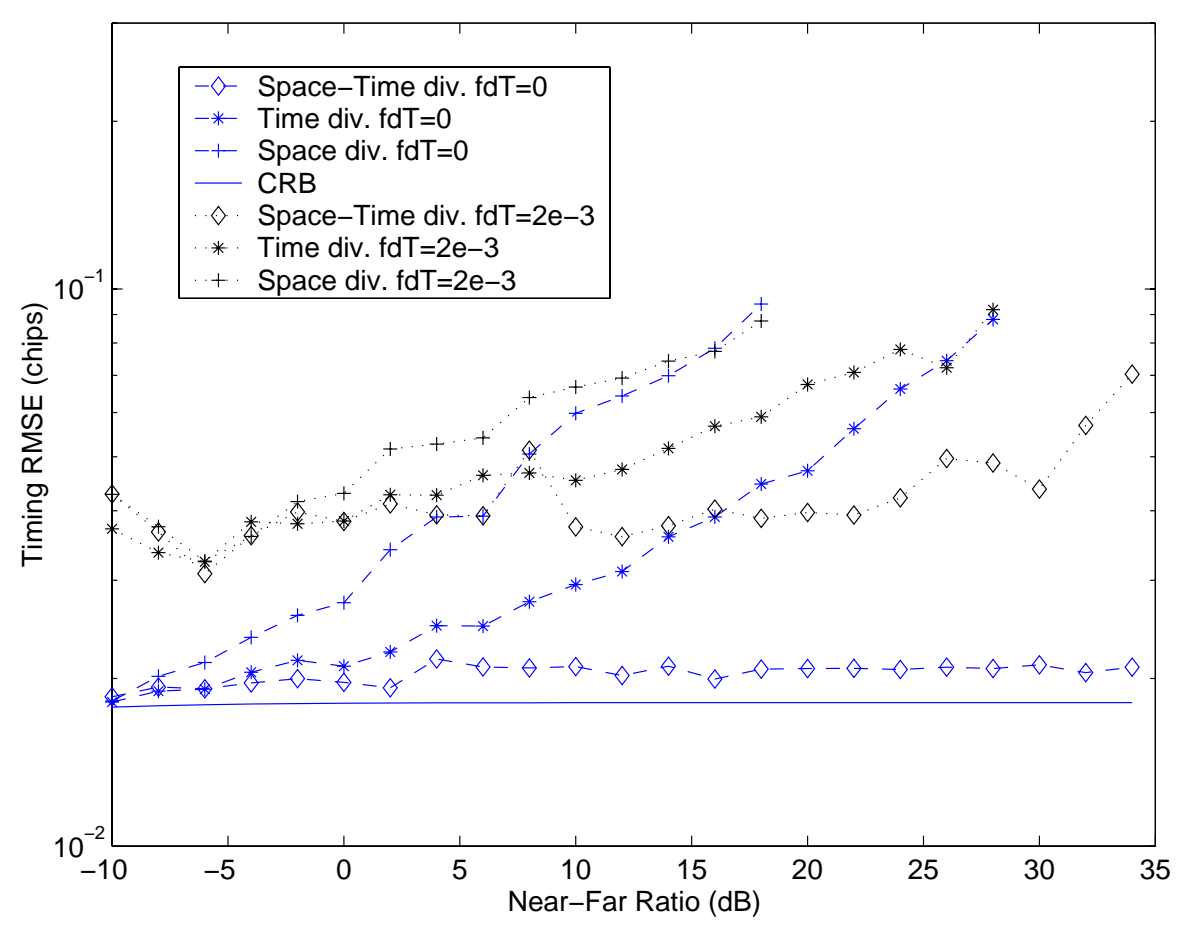

(a) RMSE

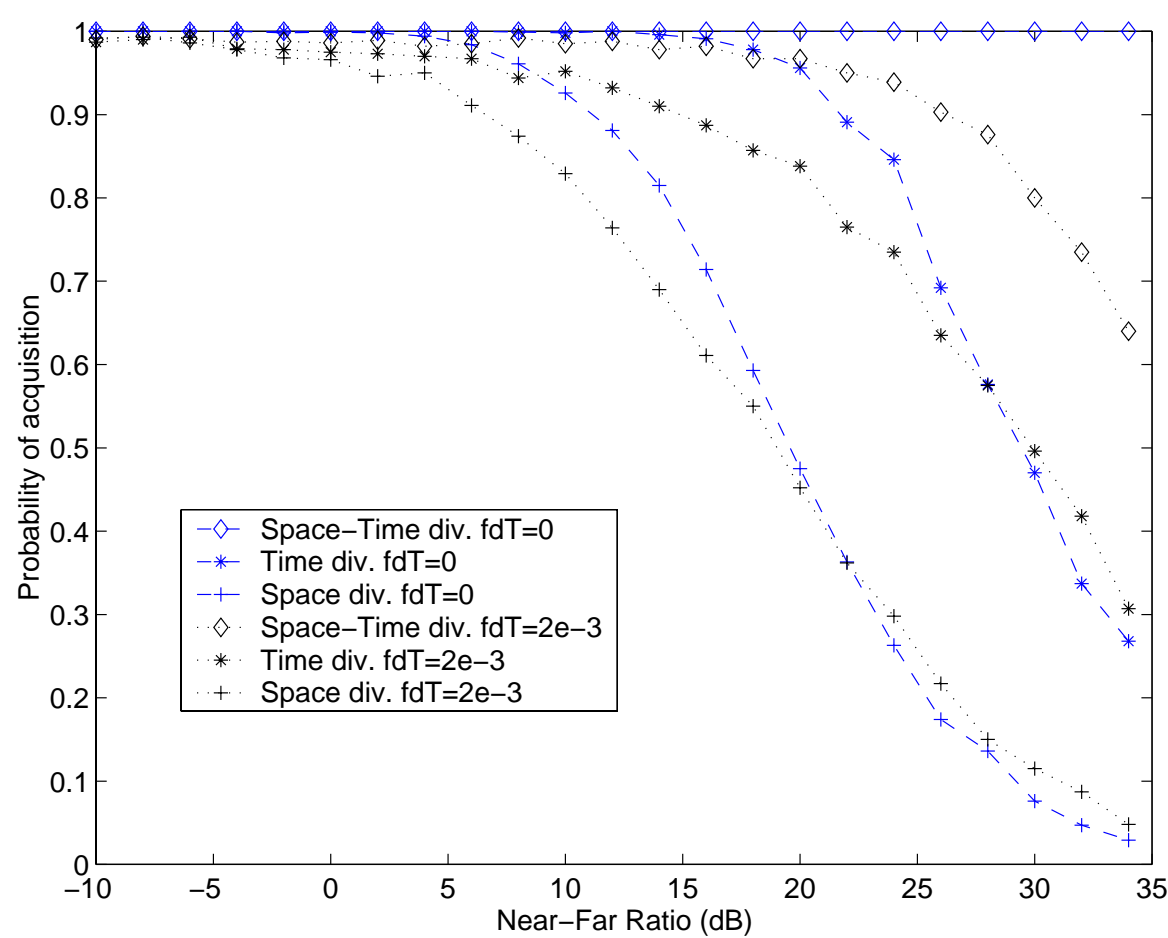

(b) Probability of acquisition

Figure 5. Performance of the STD, TD and SD estimators as a function of the near-far ratio NFR in two different channels. $M=80, K=10, N=15, L=4, \mathrm{~Eb} / \mathrm{No}=4 \mathrm{~dB}$ per antenna. 


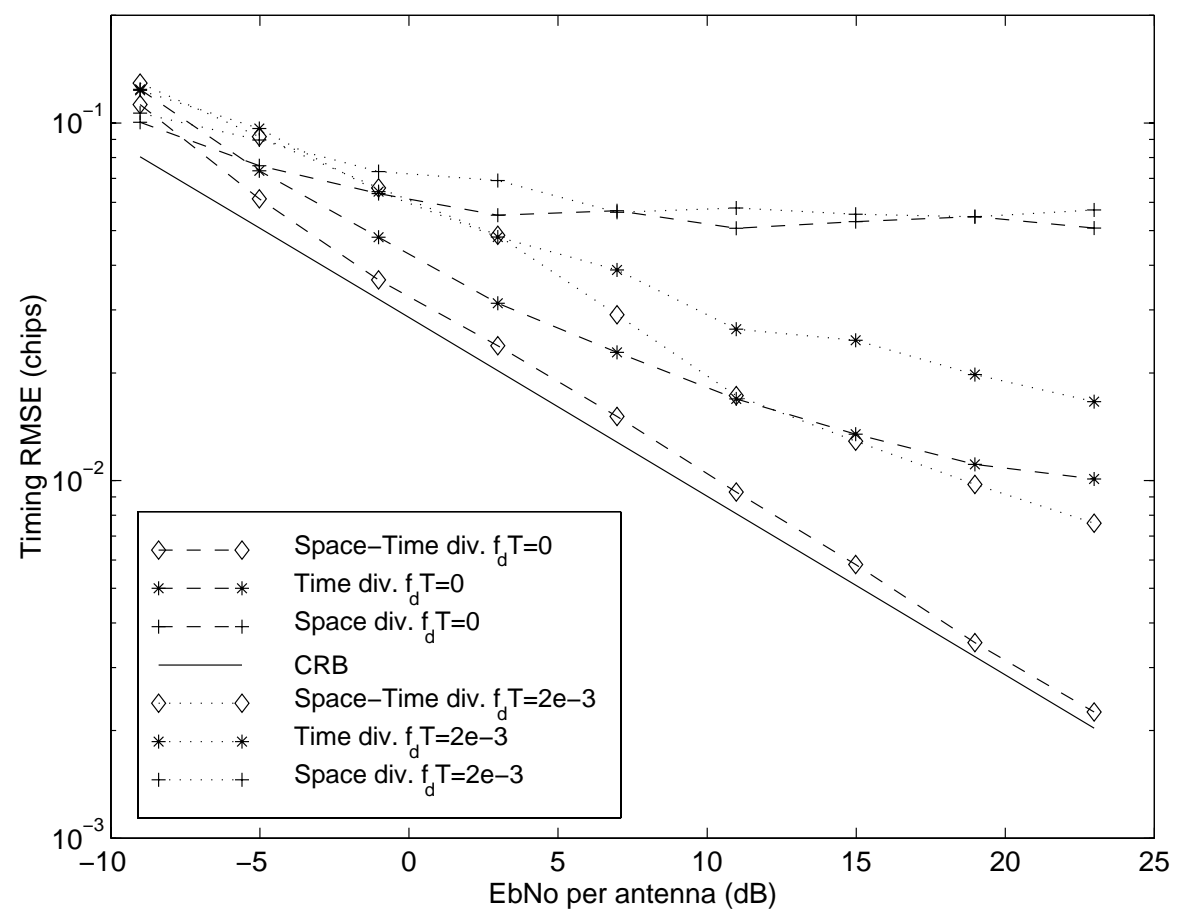

(a) RMSE

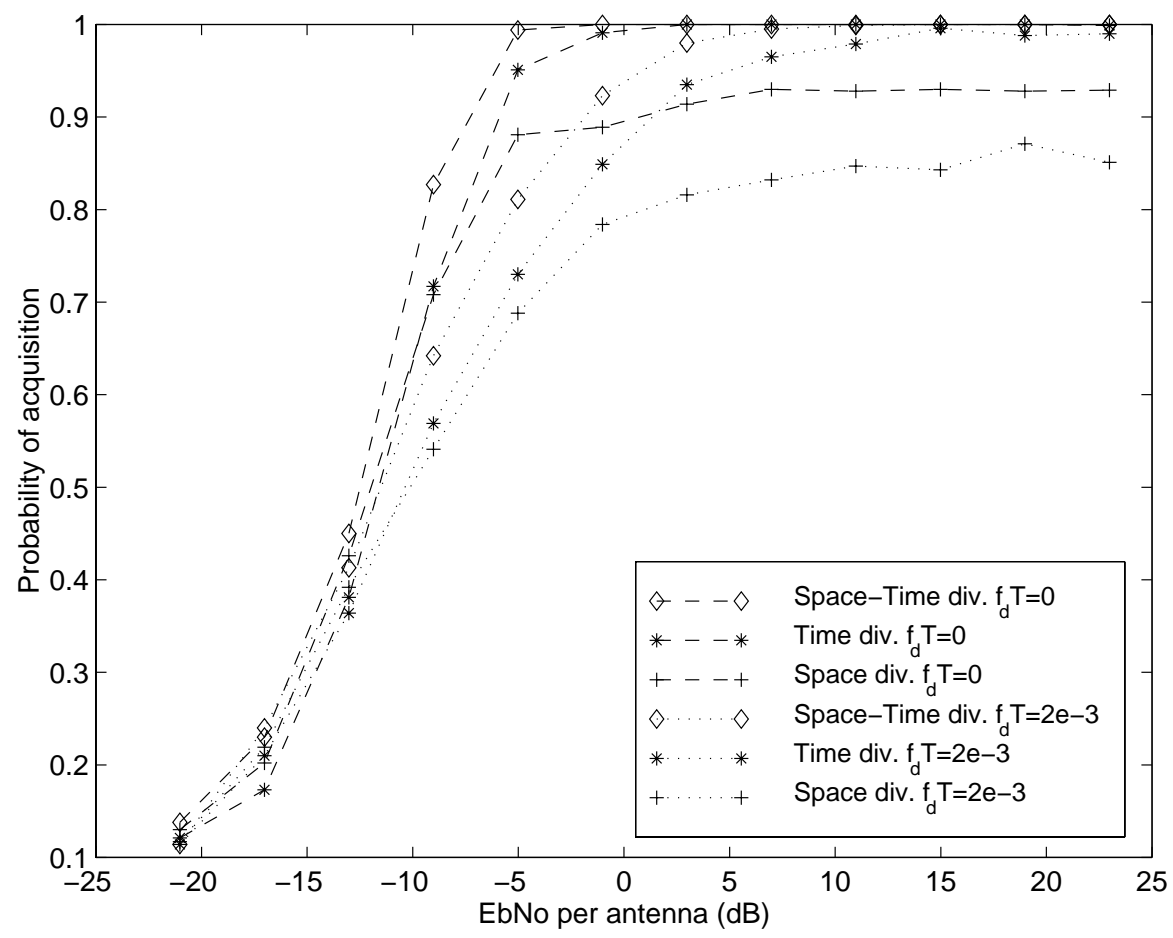

(b) Probability of acquisition

Figure 6. Performance of the STD, TD and SD estimators as a function of the Eb/No in two different channels. $M=80, K=10, N=15, L=4$, NFR $=10 \mathrm{~dB}$. 


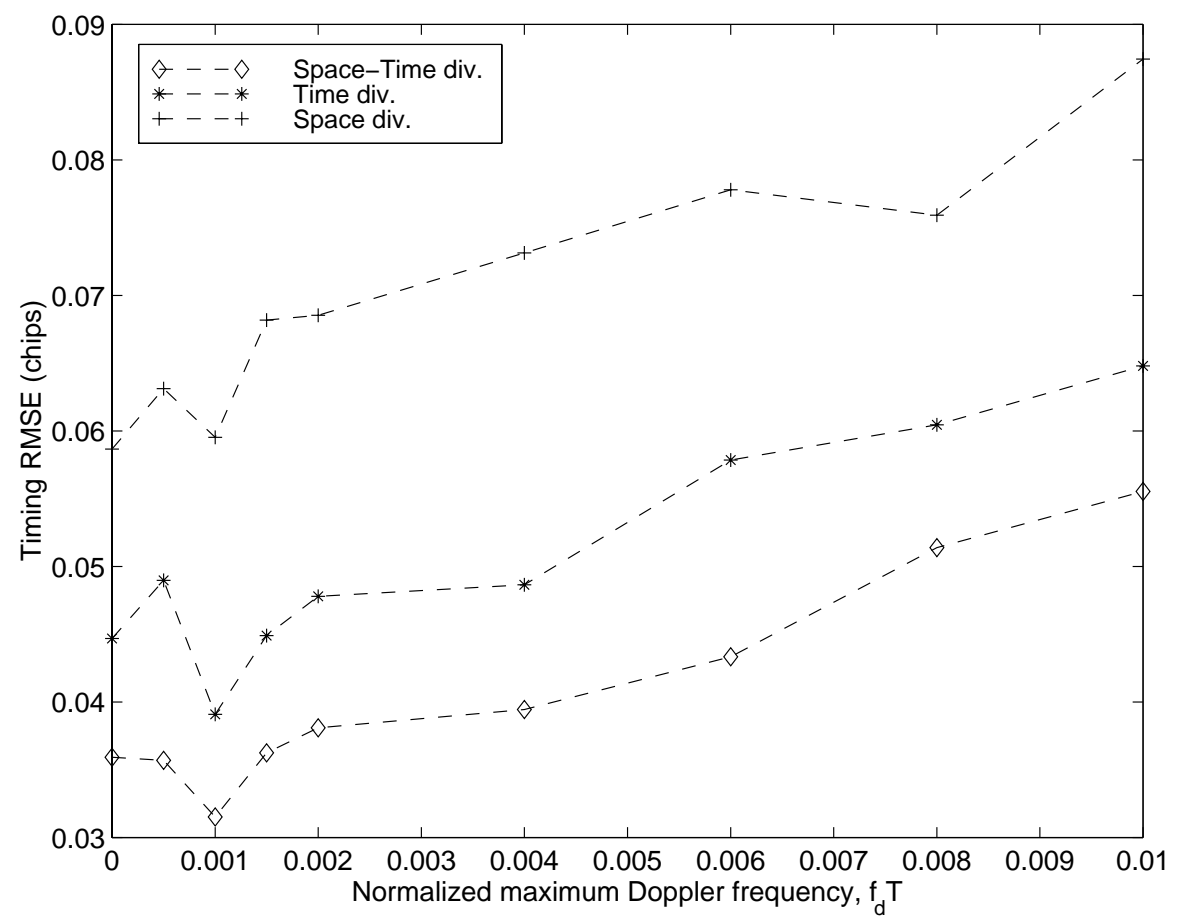

(a) RMSE

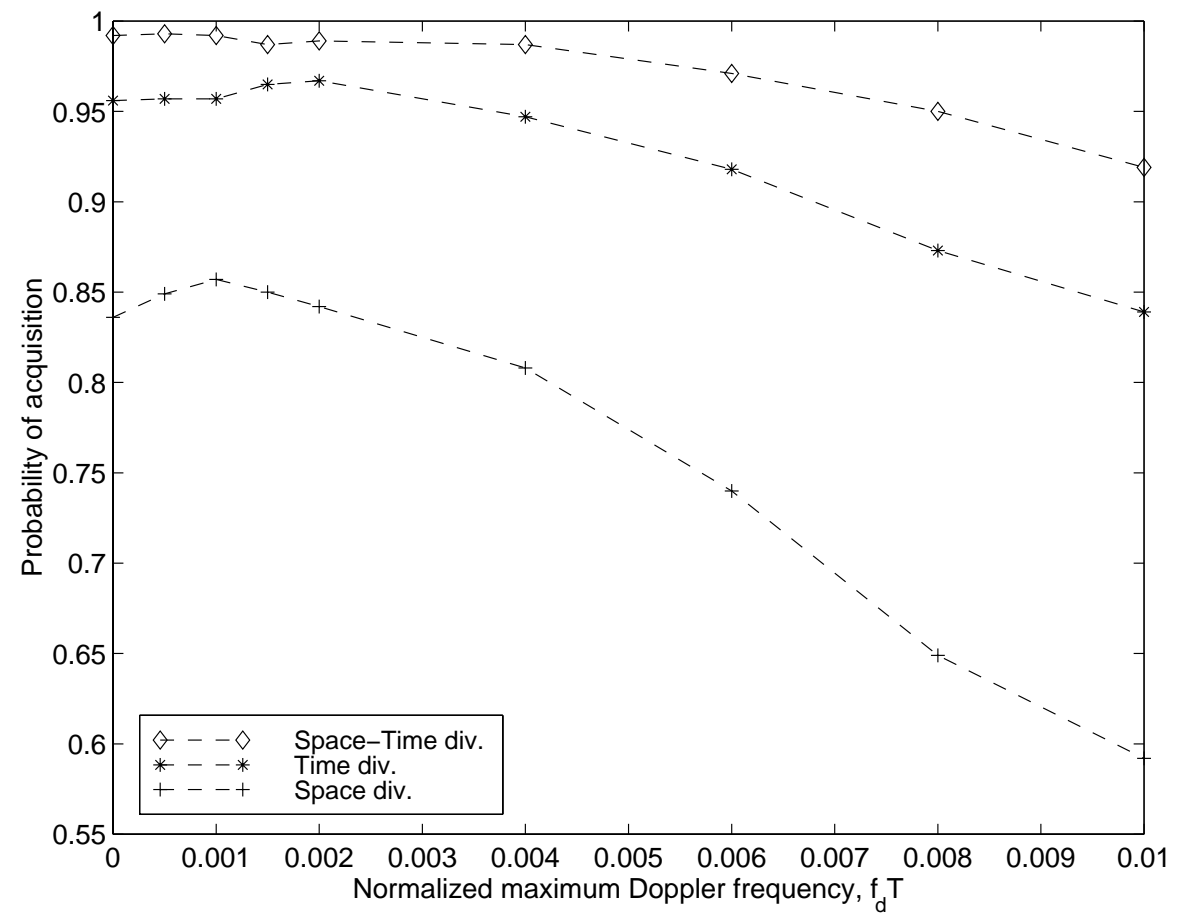

(b) Probability of acquisition

Figure 7. Performance of the STD, TD and SD estimators as a function of Doppler spread. $M=80, K=10, N=15, L=4, \mathrm{~Eb} / \mathrm{No}=4 \mathrm{~dB}$ per antenna, $\mathrm{NFR}=10 \mathrm{~dB}$. 


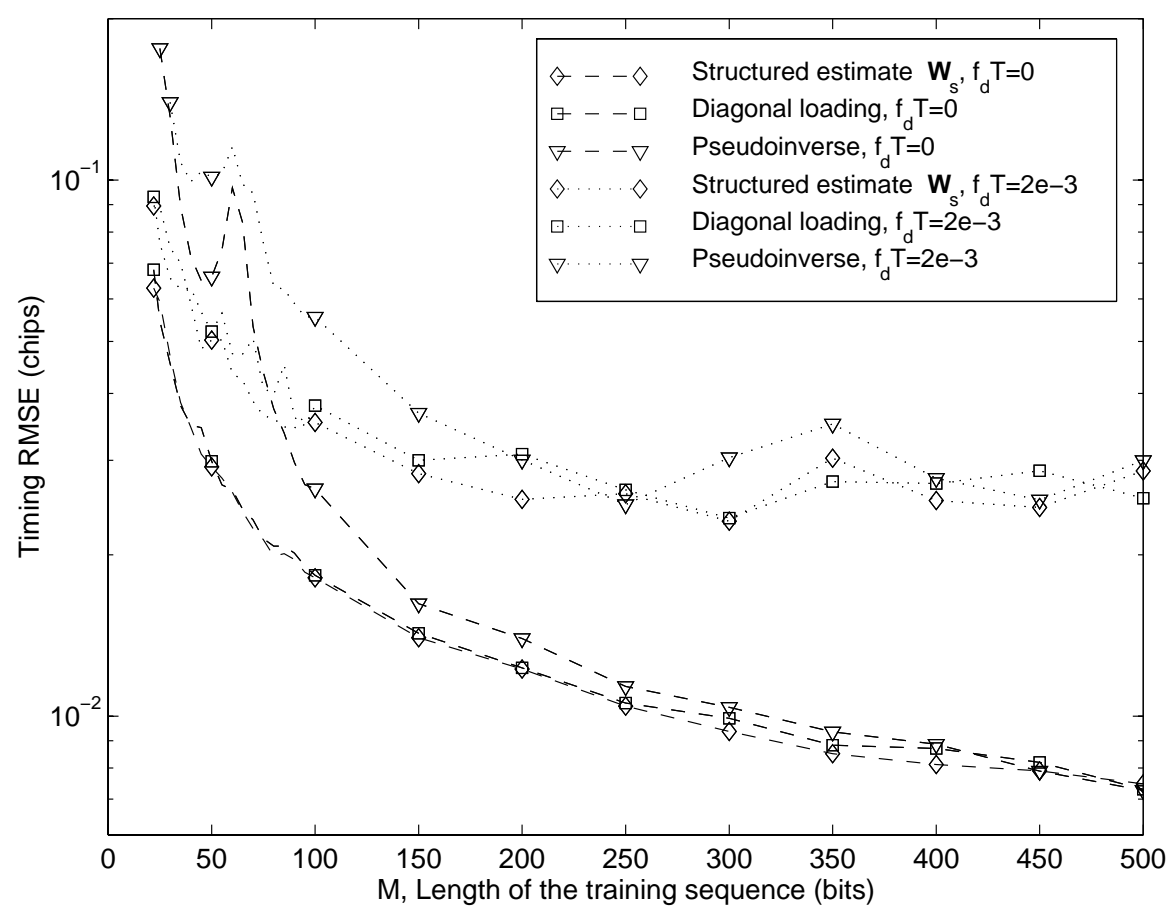

Figure 8. Comparison of the performance for three different estimates of the correlation matrix in two different channels. $K=10, N=15, L=4, \mathrm{~Eb} / \mathrm{No}=4 \mathrm{~dB}$ per antenna, $\mathrm{NFR}=10 \mathrm{~dB}, \lambda=\sigma_{w}^{2}$.

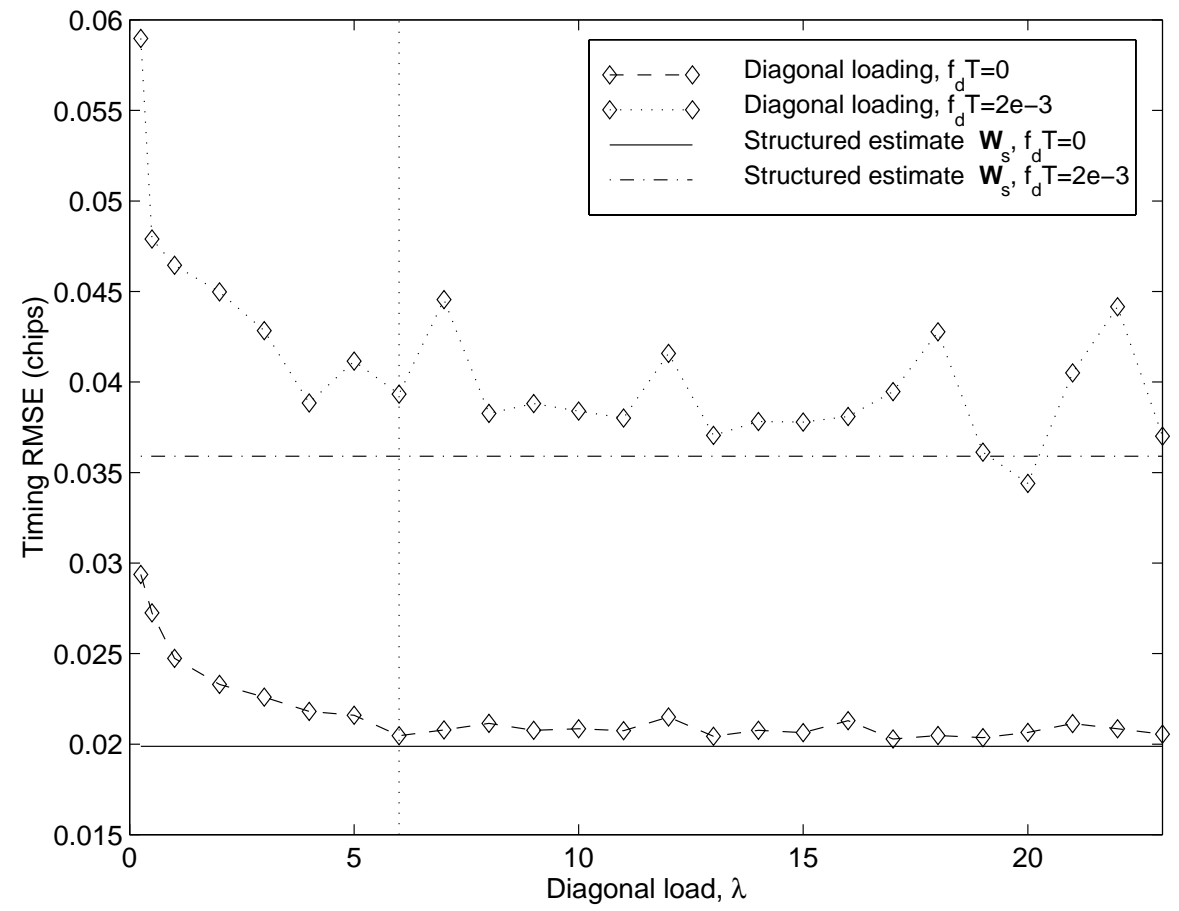

Figure 9. Effect of varying the diagonal loading factor in two different channels. The power of the white-noise is $\sigma_{w}^{2}=6$. $M=80, K=10, N=15, L=4, \mathrm{~Eb} / \mathrm{No}=4 \mathrm{~dB}$ per antenna, $\mathrm{NFR}=10 \mathrm{~dB}$ 